Fix-and-relax approaches for controlled tabular adjustment Daniel Baena Jordi Castro José A. González Dept. of Stat. and Operations Research Universitat Politècnica de Catalunya daniel.baena@upc.edu jordi.castro@upc.edu jose.a.gonzalez@upc.edu Research Report UPC-DEIO DR 2013-02 July, 2013; updated July 2014, October 2014

Report available from http://www-eio.upc.es/ jcastro 



\title{
Fix-and-relax approaches for controlled tabular adjustment
}

\author{
Daniel Baena ${ }^{a}$, Jordi Castro*,a , José A. González ${ }^{\mathrm{a}}$ \\ ${ }^{a}$ Dept. of Statistics and Operations Research, Universitat Politècnica de Catalunya, Barcelona
}

\begin{abstract}
Controlled tabular adjustment (CTA) is a relatively new protection technique for tabular data protection. CTA formulates a mixed integer linear programming problem, which is challenging for tables of moderate size. Even finding a feasible initial solution may be a challenging task for large instances. On the other hand, end users of tabular data protection techniques give priority to fast executions and are thus satisfied in practice with suboptimal solutions. This work has two goals. First, the fix-and-relax (FR) strategy is applied to obtain good feasible initial solutions to large CTA instances. FR is based on partitioning the set of binary variables into clusters to selectively explore a smaller branch-and-cut tree. Secondly, the FR solution is used as a warm start for a block coordinate descent (BCD) heuristic (approach named $\mathrm{FR}+\mathrm{BCD}$ ); $\mathrm{BCD}$ was confirmed to be a good option for large CTA instances in an earlier paper by the second and third co-authors (Computers \& Operations Research 2011). We report extensive computational results on a set of real-world and synthetic CTA instances. FR is shown to be competitive compared to CPLEX branch-and-cut in terms of quickly finding either a feasible solution or a good upper bound. FR+BCD improved the quality of FR solutions for approximately 25\% and 50\% of the synthetic and real-world instances, respectively. FR or FR+BCD provided similar or better solutions in less CPU time than CPLEX for $73 \%$ of the difficult real-world instances.
\end{abstract}

Key words: Fix-and-Relax, Block Coordinate Descent, Mixed-integer Linear Programming, Controlled Tabular Adjustment, Primal Heuristics, Feasibility Pump, Statistical Disclosure Control

\section{Introduction}

Microdata and tabular data protection are the two main disciplines of statistical disclosure control. The purpose of this field is to avoid that confidential information can be derived from data released. This is one of the main concerns of National Statistical Agencies (NSAs), which have to disseminate a large amount of information minimizing at the same time the disclosure risk of individual respondents. Tabular data is obtained by crossing two or more categorical variables in a microdata file. For each cell, the table may report either the number of individuals (frequency tables) or information about another variable (magnitude tables). More details can be found in the recent survey [5] and the monographs [26, 27].

*Corresponding address: Dept. of Statistics and Operations Research, Universitat Politècnica de Catalunya, Campus Nord, Office C5203, Jordi Girona 1-3, 08034 Barcelona, Catalonia, Spain

Email addresses: daniel . baena@upc .edu (Daniel Baena), jordi.castro@upc .edu (Jordi Castro), jose.a.gonzalez@upc .edu (José A. González) 


\begin{tabular}{c|c|c|c|c|}
\multicolumn{1}{c}{$t_{1}$} & \multicolumn{1}{c}{$t_{2}$} \\
$\vdots$ & $\ldots$ & $\ldots$ & $\ldots$ & $\ldots$ \\
\cline { 2 - 5 } $51-55$ & $\ldots$ & $38000 €$ & $40000 €$ & $\ldots$ \\
\cline { 2 - 5 } $56-60$ & $\ldots$ & $39000 €$ & $42000 €$ & $\ldots$ \\
\cline { 2 - 5 }$\vdots$ & $\ldots$ & $\ldots$ & $\ldots$ & $\ldots$ \\
\cline { 2 - 5 } & & & &
\end{tabular}

(a)

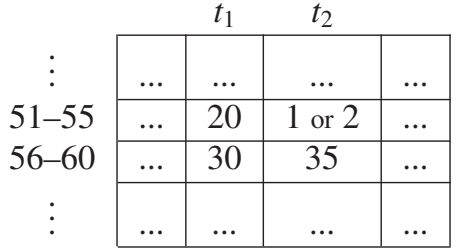

(b)

Figure 1: Example of disclosure in tabular data. (a) Average salary per age and town. (b) Number of individuals per age and town. If there is only one individual in town $t_{2}$ and age interval 51-55, then any external attacker knows the salary of this single person is $40000 €$. For two individuals, any of them can deduce the salary of the other, becoming an internal attacker.

Although cell tables report aggregated information for several respondents-so they could be considered anonymized - there is a risk of disclosing individual data. Figure 1 illustrates this situation with a simple case. The left table (a) reports the average salary of individuals by age (row variable) and town (column variable), while table (b) provides the number of individuals. If there were only one individual of age between 51-55 in town $t_{2}$, then any external attacker would know the confidential salary of this person. For two individuals, any of them could disclose the other's salary, becoming an internal attacker. Cells that require protection (such as that of the example) are named sensitive, unsafe, or confidential cells. Sensitive cells are a priori detected by some sensitivity rules. The above example showed the simplest minimum-frequency rule, which considers sensitive those cells with very few respondents. The most widely used rule, named $p-\%$ rule, considers a cell unsafe if some respondent may obtain an estimate of another respondent contribution within a $p-\%$ precision. A detailed description of these rules can be found in [27].

A tabular data protection method can be seen as a map $F$ such that $F(T)=T^{\prime}$, i.e., table $T$ is transformed to another table $T^{\prime}$. Two are the main requirements for $F$ : (1) the output table $T^{\prime}$ should be "safe", and (2) the quality of $T^{\prime}$ should be high (or equivalently, the information loss should be small), i.e., $T^{\prime}$ should be a good replacement for $T$. The disclosure risk can be analyzed through the inverse map $T=F^{-1}\left(T^{\prime}\right)$ : if not available or difficult to compute by any data attacker, then we may guarantee that $F$ is safe. Controlled Tabular Adjustment (CTA) [3, 11] is a recent technique for the protection of any tabular data. It was empirically observed in [6] that estimates $\hat{T}=\hat{F}^{-1}\left(T^{\prime}\right), \hat{F}^{-1}$ being an estimate of $F^{-1}$ for CTA, were not close to $T$ for some real tables. CTA can thus be considered a safe method in general. Moreover, the quality of CTA solutions has shown to be high [10], higher than that provided by alternative methods in some real instances [9].

The goal of CTA - which will be formulated in Section 2 - is, given a table with any structure, to find the closest safe table to the original one. This is achieved by adding the minimum amount of deviations (or perturbations) to the original cell tables that makes the released table safe. Safety is guaranteed by imposing that sensitive cells in the new protected table are far enough from the original value. This means the cell value is either above or below some certain values, thus a disjunctive constraint involving a binary variable is needed for each sensitive cell. The minimum amount of above or below perturbations required for each sensitive cell are named, respectively, upper protection and lower protection levels. Changes in sensitive cells force other changes in the remaining cells to guarantee that the value of total or marginal cells is preserved. 
Although it is a recent approach, CTA is gaining recognition among NSAs; for instance, CTA is considered a relatively new emerging method in the recent monographs [26, 27]. We recently implemented a package for CTA in collaboration with the NSAs of Germany and the Netherlands, within a project funded by Eurostat, the Statistical Office of the European Communities. This package has been largely improved within the FP7-INFRA-2010-262608 project funded by the European Union, with the participation, among others, of the national statistical institutes of Germany, Netherlands, Finland, Sweden and Slovenia. This CTA software is included in the tauArgus package [25] (http://neon.vb.cbs.nl/casc/tau.htm), used for many European national statistical institutes for the protection of tabular data. Among the recent literature on CTA variants we find [8, 24]. In recent specialized workshops on statistical disclosure control, some NSAs stated that perturbative methods, like CTA, are gaining acceptance [31], and perturbative approaches are being used for the protection of national census tables (e.g., [21] for Germany). CTA has also been used within other wider protection schemes, such as the pre-tabular protection method of [20]. In addition, some National Statistical Agencies are questioning current non-perturbative protection methods because "the task of balancing confidentiality and usability [...] is nearly impossible" [30]. Therefore there is a need for new methods, and this justifies the research on CTA and other approaches. Indeed, there is no actually any protection method that fits the needs of all NSAs in the world.

From a computational point of view, the size of the CTA optimization problem is by far smaller than for other well-known protection methods, such as the cell suppression problem [4, 19]. Despite these nice features, CTA formulates a challenging mixed integer linear problem (MILP) for current state-of-the-art solvers (such as CPLEX or XPress). Optimal (or suboptimal, e.g., with a 5\% gap) solutions may require many hours of execution for medium instances; very large or massive tables can not be tackled with current technology. Several approaches have been tried to speed up the solution time. A straightforward Benders reformulation of the problem was attempted in [7], but promising results were only obtained for two-dimensional tables (i.e., tables obtained by crossing two categorical variables, whose constraints are represented by a node-arc network incidence matrix [5]). Heuristic and metaheuristic methods were attempted in [22], but they only solved small two-dimensional and three-dimensional tables of up to 625 and 8000 cells, respectively, while we consider in this work much more complex synthetic and real tables from the literature, of up to 200000 and 36000 cells, respectively. For instance, we generated a set of 20 two-dimensional and 20 three-dimensional tables with the same characteristics (sizes and number of sensitive cells) than those in [22]. We remark that: (1) the tables used in [22] were also randomly generated; (2) the matrix constraints only depends on the table structure (two- or three-dimensional table) so they were the same in our experiments and those in [22]; (3) although the instances are not exactly the same, what makes difficult (in general) a problem is the structure of the matrix constraints and the number of sensitive cells (which is associated to the number of binary variables of the optimization problem); those characteristics are the same in our experiments and those of [22]. CPLEX 12.5 found a $0 \%$ gap solution for all these two-dimensional tables with an average CPU time of 0.02 seconds (the maximum time required by an instance was 0.03 seconds). For the three-dimensional tables, the average CPU time was 0.2 seconds (the maximum time for an instance was 0.49 seconds), again for $0 \%$ gap solutions. No CPU time comparison with CPLEX was reported in [22]; it was just stated that CPLEX 8.1 could not solve the instances. Therefore, up to now, there is no conclusive evidence that those metaheuristics are helpful for the CTA problem.

We also tried in the past other general metaheuristics as genetic algorithms without success: combinations or modifications of solutions are not expected to satisfy the large number of linear 
constraints with no particular structure of CTA. Indeed, these constraints are usually complex, and any practical approach must rely on the efficient solution of (usually difficult) linearly constrained problems (either LPs or MILPs). The approaches in this paper rely on decomposing the problem into smaller, thus tractable, MILP instances. It is worth to note that even the LPs obtained from large CTA instances by fixing the binary variables are very difficult for today stateof-the-art solvers. Indeed, some of these instances have been included in standard LP repositories [29].

The purpose of this work is twofold. Its first goal is to apply a fix-and-relax (FR) heuristic [13] to the MILP CTA problem. Briefly, FR partitions the set of binary variables into $k$ clusters, and iteratively optimizes for each cluster $i=1, \ldots, k$, fixing the binary variables of clusters $j<i$ at the optimal value found in previous iterations, and relaxing the integrality of binary variables of clusters $j>i$. The effect of this partitioning of the set of binary variables is that the nodes of the branch-and-cut tree are selectively explored. Equipping this procedure with a backward repartition strategy (details will be given in Section 3.1), if the MILP is feasible then FR will always provide a feasible, hopefully good and efficient, suboptimal solution. The approach cannot guarantee the optimal solution, but in practice end users of statistical data protection techniques prefer quick suboptimal solutions than optimal costly ones, i.e., requiring too many hours, days or weeks of CPU time.

The second objective of the work is to apply a hybrid approach combining FR and the block coordinate descent (BCD) heuristic, which was successfully applied to some classes of CTA problems in [23]. This hybrid method will be named FR+BCD. Indeed, FR is efficient for computing initial, hopefully good, feasible points, while BCD requires a feasible starting point. Therefore, both heuristics are complementary. As it will be shown in Section 4, BCD, warm started with the FR solutions, was able to reduce the gap of the FR solution in approximately half of the real-world CTA instances. In 25 of the 34 real-world instances FR or FR+BCD provided similar or better objective functions in less CPU time than the state-of-the-art MILP solver CPLEX.

FR has been successfully applied in the past mainly to scheduling problems [13, 15, 16]. In those applications, variables and constraints can naturally be partitioned according to some sequential stages, two consecutive ones being only linked by a few of the variables and constraints of each partition. Such a structure can also be found in some classes of tables, named two dimensional tables with one hierarchical variable, or, shortly, 1H2D tables. These tables are obtained by crossing a particular categorical variable with a set of, say, $h$ categorical variables that have a hierarchical relation; this results in a set of $h$ two-dimensional tables with some common cells. For instance, Figure 2 (from [5]) illustrates a particular 1H2D table. The left subtable shows number of respondents for "region" $\times$ "profession"; the middle subtable is a "zoom in" of region $R_{2}$, providing the number of respondents in municipalities of this region; finally the right subtable details the ZIP codes of municipality $R_{21}$. This type of tables, which are relevant for NSAs, are a priori suitable for FR. Most of the instances tested in the computational results of this work are $1 \mathrm{H} 2 \mathrm{D}$, and, as it will be shown, FR provides good solutions in a fraction of the time required by state-of-the-art branch-and-cut solvers (to obtain equivalent solutions, i.e., with the same objective function value). It will be seen that FR+BCD improved the FR solutions in only $25 \%$ of these $1 \mathrm{H} 2 \mathrm{D}$ tables. For real-world tables, this percentage increased up to $50 \%$, making $\mathrm{FR}+\mathrm{BCD}$ a competitive approach.

The paper is organized as follows. Section 2 outlines the MILP CTA problem. Section 3.1 describes the FR heuristic for CTA; it also outlines the BCD approach. Finally, Section 4 presents extensive computational results, showing the effectiveness of FR and FR+BCD for 


\begin{tabular}{l|ll|l|} 
& \multicolumn{1}{c}{$C_{1}$} & \multicolumn{1}{c}{$C_{2}$} & \multicolumn{1}{c}{$C_{3}$} \\
\cline { 2 - 4 }$R_{1}$ & 5 & 6 & 11 \\
$R_{2}$ & 10 & 15 & 25 \\
\cline { 2 - 4 }$R_{3}$ & 15 & 21 & 36 \\
\cline { 2 - 4 } & &
\end{tabular}

$T_{1}$

\begin{tabular}{l|ll|l|} 
& \multicolumn{1}{c}{$C_{1}$} & $C_{2}$ & \multicolumn{1}{c}{$C_{3}$} \\
\cline { 2 - 4 }$R_{21}$ & 8 & 10 & 18 \\
$R_{22}$ & 2 & 5 & 7 \\
\cline { 2 - 4 }$R_{2}$ & 10 & 15 & 25 \\
\cline { 2 - 4 } & & &
\end{tabular}

$T_{2}$

\begin{tabular}{l|ll|l|}
\multicolumn{1}{c}{} & \multicolumn{1}{c}{$C_{1}$} & \multicolumn{1}{c}{$C_{2}$} & \multicolumn{1}{c}{$C_{3}$} \\
\cline { 2 - 4 }$R_{211}$ & 6 & 6 & 12 \\
$R_{212}$ & 2 & 4 & 6 \\
\cline { 2 - 4 }$R_{21}$ & 8 & 10 & 18 \\
\cline { 2 - 4 } & & &
\end{tabular}

$T_{3}$

Figure 2: 1H2D table made of three subtables: "region" $\times$ "profession", "municipality" $\times$ "profession" and "zip code"x"profession".

synthetic $1 \mathrm{H} 2 \mathrm{D}$ and real-world tables.

\section{The MILP formulation of the CTA problem}

Any CTA problem instance can be represented by the following parameters :

- A set of cells $a_{i}, i \in \mathcal{N}=\{1, \ldots, n\}$, that satisfies $\mathcal{M}=\{1, \ldots, m\}$ linear relations $A a=b$, $a \in \mathbb{R}^{n}$ being the vector of $a_{i}$ 's, and $A \in \mathbb{R}^{m \times n}$. These linear relations impose that the set of inner cells has to be equal to the total or marginal cell, i.e., if $\mathcal{I}_{j}$ is the set of inner cells of relation $j \in \mathcal{M}$, and $t_{j}$ is the index of the total cell of relation $j$, the constraint associated to this relation is $\left(\sum_{i \in \mathcal{I}_{j}} a_{i}\right)-a_{t_{j}}=0$.

- Nonnegative cell weights $w_{i}, i \in \mathcal{N}$, used in the definition of the objective function. These weights penalize perturbations from the original cell values in the released table. Cells weights are usually a function of the cell value, e.g., $w_{i}=1 / a_{i}$-for this particular weights, the objective function represents relative cell deviations.

- A lower and upper bound for each cell $i \in \mathcal{N}$, respectively $l_{a_{i}}$ and $u_{a_{i}}$, which can be considered publicly known.

- A set $\mathcal{S}=\left\{i_{1}, i_{2}, \ldots, i_{s}\right\} \subseteq \mathcal{N}$ of indices of sensitive or confidential cells.

- A lower and upper protection level for each sensitive cell, respectively, $l p l_{i}$ and $u p l_{i}, i \in \mathcal{S}$. Values of sensitive cells must be out of the interval $\left(a_{i}-l p l_{i}, a_{i}+u p l_{i}\right)$ in the released table.

The purpose of CTA is to find the closest safe values $x_{i}$ to $a_{i}$. Considering any distance $\ell$, CTA can be formulated as

$$
\begin{array}{cl}
\min _{x} & \|x-a\|_{\ell} \\
\text { s. to } & A x=b \\
& l_{a_{i}} \leq x_{i} \leq u_{a_{i}} \quad i \in \mathcal{N} \\
& x_{i} \leq a_{i}-l p l_{i} \text { or } x_{i} \geq a_{i}+u p l_{i} \quad i \in \mathcal{S} .
\end{array}
$$

The disjunctive constraints of (1) guarantee the published value is safely out of the interval $\left(a_{i}-l p l_{i}, a_{i}+u p l_{i}\right)$. Problem (1) can also be formulated in terms of deviations from the current 
cell values. Defining $z_{i}=x_{i}-a_{i}, i \in \mathcal{N}$-and similarly $l_{z_{i}}=l_{a_{i}}-a_{i}$ and $u_{z_{i}}=u_{a_{i}}-a_{i}$-, (1) can be recast as

$$
\begin{array}{cl}
\min _{z} & \|z\|_{\ell} \\
\text { s. to } & A z=0 \\
& l_{z_{i}} \leq z_{i} \leq u_{z_{i}} \quad i \in \mathcal{N} \\
& z_{i} \leq- \text { lpl }_{i} \text { or } z_{i} \geq u p l_{i} \quad i \in \mathcal{S},
\end{array}
$$

$z \in \mathbb{R}^{n}$ being the vector of cell deviations. Using the $\ell_{1}$ or Manhattan distance and the cell weights $w_{i}$, the objective function is $\sum_{i \in \mathcal{N}} w_{i}\left|z_{i}\right|$. Since $w_{i}$ are nonnegative, splitting the vector of deviations $z$ in two nonnegative vectors $z^{+} \in \mathbb{R}^{n}$ and $z^{-} \in \mathbb{R}^{n}$, model (2) with the $\ell_{1}$ distance can thus be written as

$$
\begin{array}{cll}
\min _{z^{+}, z^{-}, y} & \sum_{i \in \mathcal{N}} w_{i}\left(z_{i}^{+}+z_{i}^{-}\right) & \\
\text {s. to } & A\left(z^{+}-z^{-}\right)=0 & \\
& 0 \leq z_{i}^{+} \leq u_{z_{i}} & i \in \mathcal{N} \backslash \mathcal{S} \\
& 0 \leq z_{i}^{-} \leq-l_{z_{i}} & i \in \mathcal{N} \backslash \mathcal{S} \\
& u p l_{i} y_{i} \leq z_{i}^{+} \leq u_{z_{i}} y_{i} & i \in \mathcal{S} \\
& l p l_{i}\left(1-y_{i}\right) \leq z_{i}^{-} \leq-l_{z_{i}}\left(1-y_{i}\right) & i \in \mathcal{S} \\
& y_{i} \in\{0,1\}, & i \in \mathcal{S},
\end{array}
$$

$y \in \mathbb{R}^{s}$ being the vector of binary variables associated to protection directions. When $y_{i}=1$ the constraints mean $u p l_{i} \leq z_{i}^{+} \leq u_{z_{i}}$ and $z_{i}^{-}=0$, thus the protection direction is "upper"; when $y_{i}=0$ we get $z_{i}^{+}=0$ and $l p l_{i} \leq z_{i}^{-} \leq-l_{z_{i}}$, thus the protection direction is "lower".

\section{Heuristic methods applied to CTA}

Model (3) is a difficult MILP even for medium size tables. Finding an optimal (or quasioptimal) solution may require many hours (even days or weeks) of execution. When the number of sensitive cells is large, the branch-and-cut scheme has shown to be inefficient, and in some cases it is even unable to provide a first feasible solution. For some massive instances-such as, e.g., those in http://www-eio.upc.es/ jcastro/huge_sdc_instances.html- the LPs obtained by fixing the value of binary variables - associated to the protection directions-are even not solvable with moderate computational resources. For example, the LPs derived from the six million cells instances of the above web address exhausted the memory of a 16 gigabytes workstation when solved with the CPLEX barrier solver. Unfortunately, the alternative simplex solver is even more prohibitive, but in terms of CPU time: interior-point algorithms have shown to be much more efficient than the simplex for the LPs derived from CTA [3, 5]. In this work we consider a FR heuristic and a hybrid FR+BCD approach for CTA. FR and BCD are, respectively, described and outlined below.

\subsection{Fix-and-relax}

FR is a decomposition method based on partitioning the set of binary variables into clusters to iteratively solve a sequence of MILPs of smaller dimension than the original problem. In those smaller MILPs only a subset of variables retain their binary constraints while the rest are either fixed or relaxed. Since only a reduced subset of (non-fixed) 0-1 variables is kept integer at each FR iteration, a computational improvement is expected. FR can both be seen as an approach for obtaining (hopefully good) initial feasible solutions and primal bounds. There are 
1. Input: Number of clusters $k \geq 1$

2. Partition $\mathcal{S}$ into $\left\{V_{1}, \ldots, V_{k}\right\}$ clusters

3. Initialize $r=1$ and solve $C T A_{F R}^{1}$

4. if $C T A_{F R}^{1}$ is infeasible, STOP

5. else Store values of binary variables of $C T A_{F R}^{1}$, set lower bound $L B$, and $r \leftarrow r+1$

6. while $r \leq k$ do

7. Solve $C T A_{F R}^{r}$

8. if infeasible, redefine the partition structure as in (5)

9. else Store optimal values of binary variables of $C T A_{F R}^{r}$, and $r \leftarrow r+1$

10. end while

11. Return $U B$ (solution of $C T A_{F R}^{k}$ ) and $L B$

Figure 3: The fix-and-relax heuristic applied to the CTA problem

other approaches for initial good solutions in MILPs, such as the feasibility pump [17], but as it will seen in Section 4 in practice FR outperformed them.

FR can be briefly stated as follows. The set of binary variables is partitioned into a finite set of clusters $\left\{V_{1}, \ldots, V_{k}\right\}$. The original MILP is then decomposed into $k$ subproblems and at each iteration one of them is solved. At first iteration (counter $r$ set to 1) the subproblem considers as binary only the variables of $V_{1}$, while the integrality of binary variables in the remaining clusters is relaxed. Continuous variables in the original MILP maintain this same status at each subproblem. Hopefully, this first subproblem will be easily solved since the cardinality of $V_{1}$ is much smaller than the number of binary variables in the original MILP. Once solved, the counter $r$ is incremented and the next subproblem is considered. At subproblem of iteration $r, k>r>1$, the binary variables of clusters $V_{i}, i<r$, are fixed to the values of optimal solutions from the previous iterations; variables of cluster $V_{r}$ are considered binary, while the integrality of variables in clusters $V_{j}, j>r$ is relaxed. The process is repeated until $r=k$. If no subproblem is infeasible, a (hopefully good) feasible solution will be available after the solution of subproblem $k$. In the particular case of CTA, the set $\mathcal{S}$ of sensitive cells is partitioned into the subsets $\left\{V_{1}, \ldots, V_{k}\right\}$, and the subproblem $r$ associated to $(3)$ - which will be referred as $\left(C T A_{F R}^{r}\right)$-is

$$
\begin{array}{lll}
\min _{z^{+}, z^{-}, y} & \sum_{i=1}^{n} w_{i}\left(z_{i}^{+}+z_{i}^{-}\right) & \\
\text {s. to } & A\left(z^{+}-z^{-}\right)=0 & \\
& 0 \leq z_{i}^{+} \leq u_{z_{i}} & i \in \mathcal{N} \backslash \mathcal{S} \\
& 0 \leq z_{i}^{-} \leq-l_{z_{i}} & i \in \mathcal{N} \backslash \mathcal{S} \\
& u p l_{i} y_{i} \leq z_{i}^{+} \leq u_{z_{i}} y_{i} & i \in \mathcal{S} \\
& l p l_{i}\left(1-y_{i}\right) \leq z_{i}^{-} \leq-l_{z_{i}}\left(1-y_{i}\right) & i \in \mathcal{S} \\
& y_{i}=\tilde{y}_{i} & i \in \bigcup_{h=1, \ldots, r-1} V_{h} \\
& y_{i} \in\{0,1\} & i \in V_{r} \\
& y_{i} \in[0,1] & i \in \bigcup_{h=r+1, \ldots, k} V_{h},
\end{array}
$$

where $\tilde{y}_{i}, i \in \cup_{h=1, \ldots, r-1} V_{h}$, are the values of binary variables found at subproblems $C T A_{F R}^{1}, \ldots$, $C T A_{F R}^{r-1}$. Although FR is a heuristic for MILP problems, it is easily switched to an optimal approach by setting $k=1$.

It is worth noting that the first subproblem $C T A_{F R}^{1}$ has two main features compared to the subsequent ones: 
- The lower bound on the objective function provided by $C T A_{F R}^{1}$ is a global lower bound of (3). On the other hand, the lower bound of subproblems $r>1$ are just local lower bounds. The lower bound reported by the FR algorithm will then be that of $C T A_{F R}^{1}$. Note that the optimal solution of $C T A_{F R}^{1}$ can be considered a lower bound of 3 only if computed with a $0 \%$ gap. However, such a gap is impractical, because the solution of $C T A_{F R}^{1}$ would take a long execution time-something to avoid, since the goal of FR is to quickly provide a decent solution. In the implementation developed, the lower bound was obtained by the CPLEX routine CPXgetbestobjval. When a problem has been solved to optimality, this routine provides the optimal solution value. Otherwise, it provides the minimum objective function value of all remaining unexplored nodes in the branch-and-cut tree.

- If $C T A_{F R}^{1}$ is infeasible, then (3) is infeasible as well. However, if some subproblem $r>1$ is infeasible it can not be concluded that (3) is infeasible; it just means that we can not fix $y_{i}=\tilde{y}_{i}$, for $i \in V_{r-1}$, at subproblem $r$. To overcome this drawback, when subproblem $r>1$ is reported as infeasible, we backtrack to problem $r-1$, modifying the partition by joining the clusters $V_{r-1}$ and $V_{r}$ as follows:

$$
\left\{\begin{array}{l}
V_{r-1} \leftarrow V_{r-1} \cup V_{r} \\
V_{i} \leftarrow V_{i+1}, i=r, \ldots, k-1 \\
k \leftarrow k-1 \\
r \leftarrow r-1 .
\end{array}\right.
$$

Note that the above repartition strategy will always provide a feasible solution if (3) is feasible. Indeed, in the worst case, if subproblem $k$ is infeasible and (5) is applied $k-$ 1 times, we will end up with a unique cluster, i.e., we will be solving (3). However, in practice, as it was observed in the computational results of Section 4 this repartition strategy was never needed in the instances tested.

An outline of the FR algorithm for CTA is shown in Figure 3

\subsection{Outline of block coordinate descent}

The BCD approach applied to CTA has been described in [23]. Briefly, it consists of a sequence of CTA subproblems, each of them optimizing the objective function over the cell deviations $z^{+}, z^{-}$and a subset of the decision variables $y$, while the remaining variables $y$ are kept fixed to some direction. Provided that we start from a feasible assignment of $y$, the method can move from a solution to another, hopefully better. Although there could be uncountable strategies to determine the subset of variables to be optimized, the set $\mathcal{S}$ is usually partitioned into $k$ clusters (or blocks) and the algorithm iterates through them. However, BCD could perform indefinitely, starting again with the same or with another partition. Stopping criteria normally employed are: only one cycle of $k$ clusters; a time limit, or a specified number of subproblems without improvement in the objective function. Since the method does not account for dual information there are no means to compute a gap for the solution. Despite this, the results of [23] showed that $\mathrm{BCD}$ reaches sub-optimal but still good solutions in significantly less time than branch-and-cut schemes. The algorithm is summarized in Figure 4

Experience with BCD has shown that, in general, the performance of the method improves as the number of blocks decreases, and two blocks seems to be the best choice. Notice that one block would lead the method to a plain branch-and-cut, which might be computationally prohibitive. It has been observed that many tables are (sub-optimally) protected through manipulation of half 
1. Input: Number of clusters $k \geq 1$

2. Set feasible initial values to $y$; initialize outer iteration counter: $t \leftarrow 0$

3. while stopping criterion not satisfied and $t \leq t_{\max }$ do

4. Set inner iteration counter: $i \leftarrow 0$; divide $y$ into $k$ blocks: $y=\left\{y^{1, i}, \ldots, y^{k, i}\right\}$

5. while $i<k$ do

6. Solve (3) with respect to block $y^{i, i}$ fixing $y^{j, i}$ for $j \neq i$ : obtain $\left(y^{i, i}\right)^{*}$

7. $y^{i, i+1} \leftarrow\left(y^{i, i}\right)^{*} ; y^{j, i+1} \leftarrow y^{j, i}$ for $j \neq i$

8. $\quad i \leftarrow i+1$

9. end while

10. $t \leftarrow t+1$

11. end while

12. Return the best solution found

Figure 4: The block coordinate descent heuristic for the CTA problem

of their sensitive cells in a fraction of the time needed if the whole set of sensitive cells was considered (this fraction of time being significantly less than $1 / 2$ ).

Many tests indicate that rebuilding the partition of blocks at each iteration is clearly preferable to keep some pre-determined division. Actually, the best performances are obtained with a random division of the binary variables into blocks; this is the main strategy considered.

A disadvantage of BCD is the need to find a feasible initial assignment of directions to start the process (step 2 of algorithm of Figure 4), which may be in itself a difficult problem for large CTA instances. The heuristic approach considered in [23], which relies on the Boolean Satisfiability problem, only focuses on the constraints, and then it may provide poor quality solutions. Since FR solutions take into account the objective function, we can use them as a good warm start to BCD. This approach, named FR+BCD, will be computationally tested and seen as a very efficient option in Subsection 4.3

\section{Computational results}

The FR and FR+BCD heuristics for CTA have been coded in $\mathrm{C}++$, using the state-of-the-art CPLEX 12.5 branch-and-cut solver for the solution of subproblems (4). FR and FR+BCD were compared with the direct solution of (3) through plain CPLEX branch-and-cut, which will be referred as $\mathrm{BC}$.

All the runs were carried out on a Dell PowerEdge 6950 server with four dual core AMD Opteron $82223.0 \mathrm{GHZ}$ processors (without exploitation of parallelism capabilities) and $64 \mathrm{~GB}$ of RAM. Default values were used for the CPLEX parameters, unless explicitly stated. For the computational tests we considered a set of real-world general and synthetic 1H2D tables. Realworld general tables are standard instances used in the literature [5]. It is worth noting that some real-world instances were not included in this set since they are too difficult for both heuristic and exact MILP approaches—no feasible solution was obtained within the time limit. Synthetic instances were obtained with a generator of $1 \mathrm{H} 2 \mathrm{D}$ tables. This generator is governed by several parameters, as, for instance, the number of rows in a subtable; the number of columns per subtable; the depth of the hierarchical tree; the minimum and maximum number of rows with hierarchies for each subtable; and the probability for a cell to be marked as sensitive. The 1H2D table generator is available from http://www-eio.upc.es/ jcastro/generators_csp.html. We 


\begin{tabular}{lrrrr}
\hline instance & $\bar{n}$ & $\bar{s}$ & $\bar{m}$ & $\overline{n z}$ \\
\hline \multicolumn{5}{c}{ Symmetric } \\
\hline sym-40-50nces \\
sym-40-50-15 & 29039 & 1421 & 1334 & 58793 \\
sym-40-50-30 & 291753 & 46612 & 1388 & 64219 \\
sym-40-60-5 & 36990 & 8556 & 1336 & 58997 \\
sym-40-60-15 & 34026 & 5011 & 1521 & 74835 \\
sym-40-60-30 & 38040 & 11207 & 1539 & 68906 \\
sym-50-50-5 & 40637 & 1989 & 1562 & 81983 \\
sym-50-50-15 & 39596 & 5815 & 1541 & 79907 \\
sym-50-50-30 & 38097 & 11190 & 1512 & 76908 \\
sym-50-60-5 & 45555 & 2237 & 1662 & 91964 \\
sym-50-60-15 & 44457 & 6550 & 1644 & 89768 \\
sym-50-60-30 & 45835 & 13507 & 1666 & 92525 \\
\hline \multicolumn{5}{c}{ Asymmetric instances } \\
\hline asym-40-50-5 & 125661 & 6157 & 5677 & 254483 \\
asym-40-50-15 & 126844 & 18646 & 5700 & 256850 \\
asym-40-50-30 & 127000 & 37338 & 5703 & 257162 \\
asym-40-60-5 & 151166 & 7431 & 6321 & 306114 \\
asym-40-60-15 & 149641 & 22069 & 6296 & 303064 \\
asym-40-60-30 & 150711 & 44454 & 6314 & 305203 \\
asym-50-50-5 & 162561 & 7966 & 6400 & 328284 \\
asym-50-50-15 & 159766 & 23487 & 6346 & 322694 \\
asym-50-50-30 & 160171 & 47094 & 6354 & 323503 \\
asym-50-60-5 & 191503 & 9415 & 6982 & 386789 \\
asym-50-60-15 & 189718 & 27982 & 6953 & 383218 \\
asym-50-60-30 & 188742 & 55676 & 6937 & 381266 \\
\hline
\end{tabular}

Table 1: Characteristics of symmetric/asymmetric synthetic 1H2D instances.

fixed all parameters, but three: the number of rows per subtable ( $r \in\{40,50\})$, the number of columns per subtable $(c \in\{50,60\})$ and the percentage of sensitive cells $(s \in\{5,15,30\})$.

We considered either symmetric and asymmetric instances, i.e., instances where $u_{a_{i}}=l_{a_{i}}$ for all $i \in \mathcal{N}$ and $u_{a_{i}} \neq l_{a_{i}}$ for some $i \in \mathcal{N}$, respectively. Asymmetric instances were obtained by considering $u_{a_{i}}=\mathrm{a} \cdot l_{a_{i}}$ for all $i \in \mathcal{N}$, where a $\in\{2,5,10\}$ is the asymmetry parameter. For each combination of parameters we generated a sample of five instances varying the random generator seed. This amounted to 12 and 36 samples of five instances each one, for symmetric and asymmetric instances respectively. Although the asymmetry parameter slightly affects to the difficulty of the problem, both symmetric and asymmetric instances will be grouped by $r$, $c$ and $\mathrm{s}$ to simplify the exposition. The reported computational results are thus averaged on five and 15 replications for symmetric and asymmetric tables, respectively.

Table 1 reports the characteristics of each set of symmetric/asymmetric 1H2D instances: the average number of cells (" $" \bar{n} ")$, the average number of sensitive cells (" $\vec{s}$ "), the average number of table relations (" $m$ ") and the average number of coefficients in linear constraints (" $\overline{n z}$ "). Hierarchical synthetic tables are identified by the particular combination of parameters, i.e., sym-r-c-s for symmetric instances and asym-r-c-s for asymmetric ones. Table 2 reports the same information for real-world tables, though in this case the dimensions are not averaged. The dimensions of the MILP problems (3) are $2 n$ continuous variables, $s$ binary variables, and $m+4 s$ linear constraints.

\subsection{Tuning the number of clusters in fix-and-relax}

The performance of FR depends on the number of clusters $k$ considered. We performed an empirical study of the effect of $k$ on two particular metrics: the CPU time and the quality of the solutions provided by FR. This empirical analysis was done considering values $k \in \mathcal{K}=$ 


\begin{tabular}{|c|c|c|c|c|}
\hline instance & $n$ & $s$ & $m$ & $n z$ \\
\hline australia_ABS & 24420 & 918 & 274 & 13224 \\
\hline bts4 & 36570 & 2260 & 36310 & 136912 \\
\hline cbs & 11163 & 2467 & 244 & 22326 \\
\hline dale & 16514 & 4923 & 405 & 33028 \\
\hline destatis & 5940 & 621 & 1464 & 18180 \\
\hline hier13d4 & 18969 & 2188 & 47675 & 143953 \\
\hline hier13 & 2020 & 112 & 3313 & 11929 \\
\hline hier13x13x13a & 2197 & 108 & 3549 & 11661 \\
\hline hier $13 \times 13 \times 13 b$ & 2197 & 108 & 3549 & 11661 \\
\hline hier $13 \times 13 \times 13 c$ & 2197 & 108 & 3549 & 11661 \\
\hline hier $13 \times 13 \times 13 d$ & 2197 & 108 & 3549 & 11661 \\
\hline hier $13 \times 13 \times 13 e$ & 2197 & 112 & 3549 & 11661 \\
\hline hier13x13x7d & 1183 & 75 & 1443 & 5369 \\
\hline hier13x7x7d & 637 & 50 & 525 & 2401 \\
\hline hier16 & 3564 & 224 & 5484 & 19996 \\
\hline hier16x16x16a & 4096 & 224 & 5376 & 21504 \\
\hline hier16x16x16b & 4096 & 224 & 5376 & 21504 \\
\hline hier16x16x16c & 4096 & 224 & 5376 & 21504 \\
\hline hier16x16x16d & 4096 & 224 & 5376 & 21504 \\
\hline hier16x16x16e & 4096 & 224 & 5376 & 21504 \\
\hline nine $5 \mathrm{~d}$ & 10733 & 1661 & 17295 & 58135 \\
\hline osorio & 10201 & 7 & 202 & 20402 \\
\hline sbs2008_C & 4212 & 1135 & 2580 & 13806 \\
\hline sbs2008_E & 1430 & 382 & 991 & 4680 \\
\hline table1 & 1584 & 146 & 510 & 4752 \\
\hline table3 & 4992 & 517 & 2464 & 19968 \\
\hline table4 & 4992 & 517 & 2464 & 19968 \\
\hline table 5 & 4992 & 517 & 2464 & 19968 \\
\hline table6 & 1584 & 146 & 510 & 4752 \\
\hline table7 & 624 & 17 & 230 & 1872 \\
\hline table 8 & 1271 & 3 & 72 & 2542 \\
\hline targus & 162 & 13 & 63 & 360 \\
\hline toy3dsarah & 2890 & 376 & 1649 & 9690 \\
\hline two5in6 & 5681 & 720 & 9629 & 34310 \\
\hline
\end{tabular}

Table 2: Characteristics of real instances. 
$\{3,5,7,10,20,30,40,50\}$, and using a subset of asymmetric 1H2D instances. For each combination of parameters $r-c-s$ we considered a sample of three instances.

Each instance was solved $|\mathcal{K}|$ times, randomly partitioning the set $\mathcal{S}$ of sensitive cells into $k \in \mathcal{K}$ subsets. The stopping criterion for all the runs, i.e., subproblems (4), was a $5 \%$ optimality gap, which is computed by CPLEX as (UB - LB)/(|UB| $+10^{-10}$ ), where UB is the best integer solution (upper bound) and LB is the best achievable value from the current branch-and-cut tree (lower bound).

Figure 5 reports the CPU time (in seconds, averaged for the three instances of each sample) used by FR for the different $k \in \mathcal{K}$ number of clusters. Clearly, the CPU time increases with $k$, and the heuristic becomes prohibitive if the number of clusters is large.

The second metric, the quality of the solutions, was evaluated using the performance profile proposed in [14]. Quality was measured as the value of the objective function (thus, the lower, the better). Let $Q_{t k}$ be the quality of the solution of instance $t$ solved by FR with $k$ clusters. Note that $Q_{t k}$ for CTA is always strictly positive. The performance ratio is thus defined as

$$
v(t, k)=\frac{Q_{t, k}}{\min \left\{Q_{t, k}: k \in \mathcal{K}\right\}},
$$

i.e., the ratio between the quality of the solution obtained when instance $t$ is solved by FR with $k$ clusters over the strategy with the best (minimum) performance for this instance. The (cumulative) distribution function $P_{k}(q):[1, \infty) \rightarrow[0,1]$ is defined as

$$
P_{k}(q)=\frac{|\{t \in \mathcal{T}: v(t, k) \leq q\}|}{|\mathcal{T}|}, q \geq 1 .
$$

where $\mathcal{T}$ is the set of instances. Figure 6 shows the performance profiles for the different $k \in \mathcal{K}$. $P_{k}(q)=1$ means FR with $k$ clusters is able to solve all the instances within a factor $q$ of the best possible ratio. In our case $k=3$ is the first strategy to converge to 1 for $q \approx 1.45$ (i.e., FR with 3 blocks solves all the instances within a factor $\approx 1.45$ of the best ratio). It can also be observed that $k=3$ provides the highest quality for $80 \%$ of the instances $\left(P_{3}(1) \approx 0.8\right)$.

\subsection{Comparison between fix-and-relax and plain branch-and-cut}

From the discussion of previous Subsection, $k=3$ was set for FR. An optimality gap of 5\% was considered for all the optimization problems, either (3) or FR subproblems (4). The time limit was set to two hours for both 1H2D and real-world instances. Note that FR subproblems are also solved by CPLEX branch-and-cut; therefore the comparison is between whether using or not the FR scheme. We will refer to these two variants as FR and BC.

Tables 3 and 4 report an exhaustive comparison between FR and BC for synthetic 1H2D and real-world instances, respectively. These tables report the FR CPU time (columns " $T_{F R}$ "); the primal gap of the solution reported by FR (columns " $G A P_{F R} \%$ "); the primal gap of the solution reported by $\mathrm{BC}$ after $T_{F R}$ seconds of CPU time (columns " $G A P_{B C} \%$ "), i.e., using the same time than FR; the difference between both primal gaps (columns " $\triangle(B C, F R)$ "); the primal gap and CPU time needed by BC to compute a better solution than the feasible solution

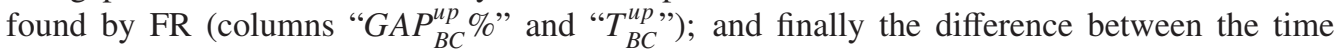
needed by BC to improve the FR solution and the time needed by FR to compute that solution (columns" $\triangle\left(T_{F R}, T_{B C}^{u p}\right)$ "). Positive values at column $\triangle(B C, F R)$ means that FR achieved a better solution than BC in the same CPU time. 


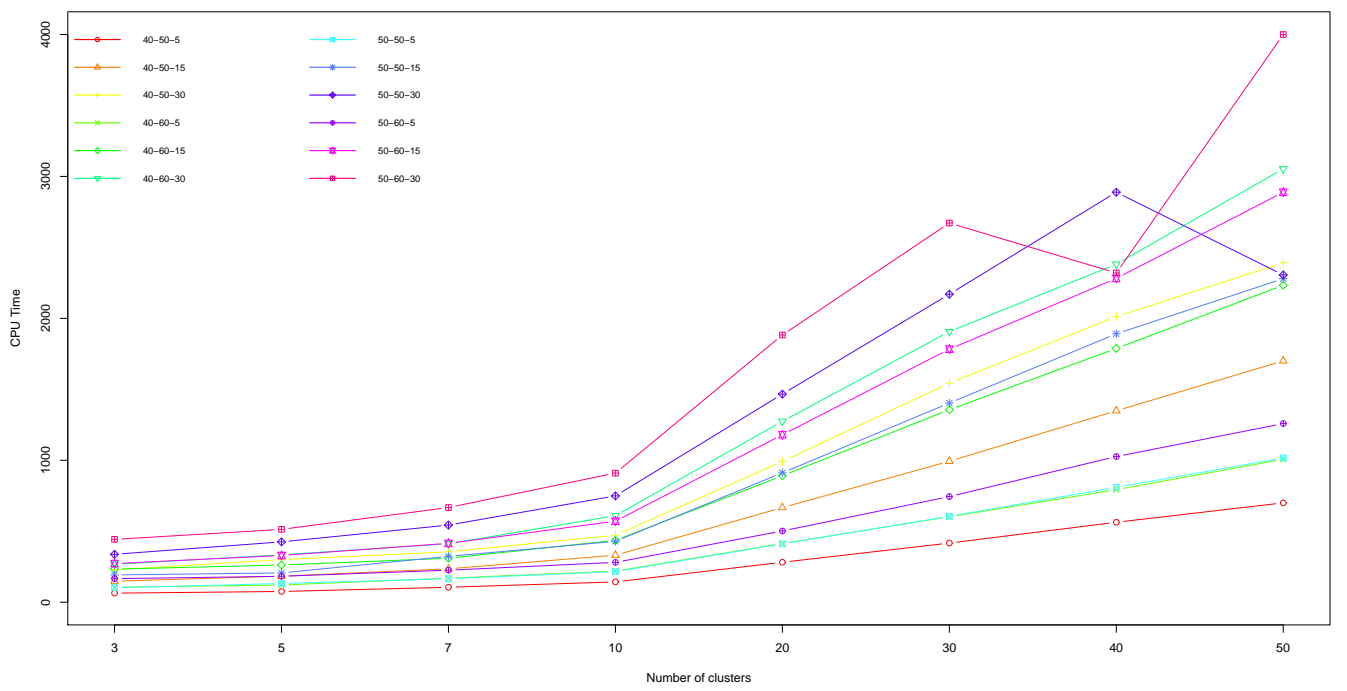

Figure 5: CPU time for different number of clusters

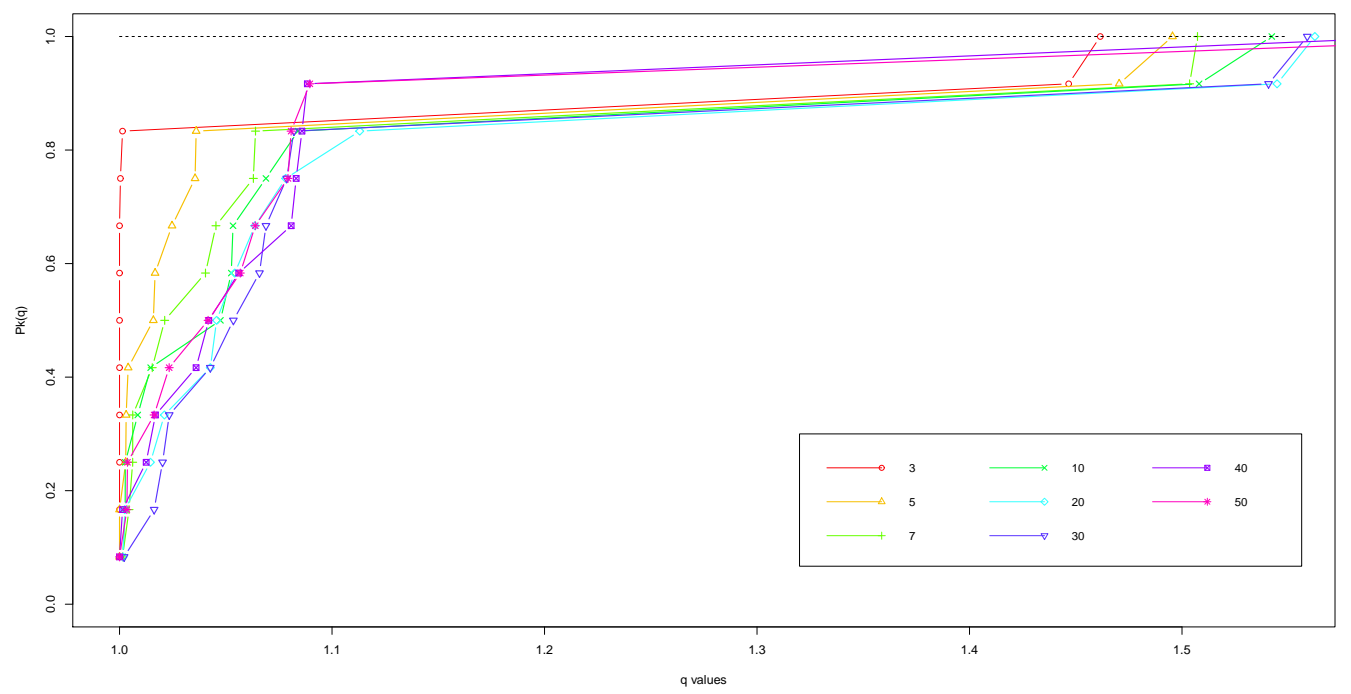

Figure 6: Performance profile of the quality of the solution for different number of clusters 


\begin{tabular}{|c|c|c|c|c|c|c|c|}
\hline instance & $T_{F R}$ & $G A P_{F R} \%$ & $G A P_{B C} \%$ & $\Delta(B C, F R)$ & $G A P_{B C}^{u p} \%$ & $T_{B C}^{u p}$ & $\Delta\left(T_{B C}^{u p}, T_{F R}\right)$ \\
\hline asym-40-50-5 & 72.76 & 2.52 & $\dagger^{(40.70,5)}$ & $+(38.18,5)$ & $f^{(1.20,13)}$ & $\ddagger^{(87.76,13)}$ & $t^{(15.00,13)}$ \\
\hline asym-40-50-15 & 158.75 & 3.55 & 86.67 & 83.11 & $\ddagger^{(1.94,13)}$ & $\ddagger^{(465.84,13)}$ & $\ddagger^{(307.09,13)}$ \\
\hline asym-40-50-30 & 210.51 & 5.60 & 99.98 & 94.38 & 1.84 & 1083.47 & 872.96 \\
\hline asym-40-60-5 & 95.40 & 2.40 & $\dagger^{(0.88,5)}$ & $\dagger^{(-1.51,5)}$ & $\ddagger^{(1.08,14)}$ & $\ddagger^{(124.01,14)}$ & $\ddagger^{(28.61,14)}$ \\
\hline asym-40-60-15 & 193.37 & 3.17 & 99.96 & 96.79 & $\ddagger^{(1.69,12)}$ & $\ddagger^{(945.40,12)}$ & $\ddagger^{(752.03,12)}$ \\
\hline asym-40-60-30 & 314.96 & 5.26 & 99.97 & 94.71 & 2.43 & 1107.84 & 792.88 \\
\hline asym-50-50-5 & 110.54 & 1.82 & $\dagger^{(0.08,8)}$ & $\dagger^{(-1.74,8)}$ & $\ddagger^{(0.62,14)}$ & $\ddagger^{(135.65,14)}$ & $\ddagger^{(25.11,14)}$ \\
\hline asym-50-50-15 & 186.95 & 3.11 & 86.66 & 83.54 & $t^{+}(1.02,11)$ & $\ddagger^{+}(804.02,11)$ & $\ddagger^{(617.07,11)}$ \\
\hline asym-50-50-30 & 333.50 & 5.94 & 99.94 & 93.99 & 2.21 & 1704.06 & 1370.55 \\
\hline asym-50-60-5 & 153.14 & 1.23 & $\dagger^{(0.45,9)}$ & $\dagger^{(-0.78,9)}$ & $\ddagger^{(0.85,13)}$ & $\ddagger^{(163.61,13)}$ & $\ddagger^{(10.46,13)}$ \\
\hline asym-50-60-15 & 282.79 & 3.05 & 93.33 & 90.28 & $\ddagger^{+}(1.46,13)$ & $\ddagger^{+}(1569.41,13)$ & $\ddagger^{(1286.62,13)}$ \\
\hline asym-50-60-30 & 406.42 & 5.52 & 99.92 & 94.40 & 2.39 & 1396.89 & 990.47 \\
\hline sym-40-50-5 & 8.99 & 2.43 & 12.69 & 10.26 & $\ddagger^{(1.61,2)}$ & $\ddagger^{(15.09,2)}$ & $\neq(6.10,2)$ \\
\hline sym-40-50-15 & 115.34 & 4.31 & 45.79 & 41.48 & $\ddagger^{(2.56,4)}$ & $\ddagger^{+}(443.91,4)$ & $\ddagger^{(328.57,4)}$ \\
\hline sym-40-50-30 & 371.35 & 4.61 & 63.90 & 59.29 & $\ddagger^{(4.36,3)}$ & $\ddagger^{(2681.80,3)}$ & $\ddagger^{(2310.45,3)}$ \\
\hline sym-40-60-5 & 10.52 & 2.79 & 14.44 & 11.65 & 1,33 & 37.76 & 27.24 \\
\hline sym-40-60-15 & 102.29 & 2.20 & 82.23 & 80.03 & $\ddagger^{(-, 0)}$ & $\ddagger^{(-, 0)}$ & $\ddagger^{(-, 0)}$ \\
\hline sym-40-60-30 & 800.45 & 4.39 & 12.88 & 8.49 & $\dagger^{(4.59,3)}$ & $\dagger^{(2870.02,3)}$ & $\dagger^{(2069.57,3)}$ \\
\hline sym-50-50-5 & 25.47 & 1.94 & $\dagger^{(12.20,3)}$ & $\dagger(10.25,3)$ & $\ddagger^{(0.72,5)}$ & $\ddagger^{(50.75,5)}$ & $\ddagger^{(25.27,5)}$ \\
\hline sym-50-50-15 & 166.33 & 3.66 & 12.74 & 9.08 & $\ddagger^{(1.95,2)}$ & $\ddagger^{(1434.04,2)}$ & $\ddagger^{(1267.71,2)}$ \\
\hline sym-50-50-30 & 511.19 & 3.45 & 66.81 & 63.36 & $\ddagger^{+}(3.71,2)$ & $\neq^{+}(5049.30,2)$ & $t^{+}(4538.11,2)$ \\
\hline sym-50-60-5 & 56.80 & 1.61 & $\dagger^{(54.33,4)}$ & $+(52.72,4)$ & $\psi^{+}(0.97,4)$ & $+(104.60,4)$ & $\ddagger^{(47.80,4)}$ \\
\hline sym-50-60-15 & 279.63 & 2.47 & 13.60 & 11.14 & $\ddagger^{(0.70,1)}$ & $\ddagger^{(1055.10,1)}$ & $\ddagger^{7}(775.47,1)$ \\
\hline sym-50-60-30 & 833.45 & 3.95 & 47.62 & 43.66 & $\ddagger^{(4.38,2)}$ & $\ddagger^{(3863.53,2)}$ & $\ddagger^{(3030.08,2)}$ \\
\hline
\end{tabular}

Table 3: Comparison between fix-an-relax and plain branch-and-cut for synthetic asymmetric and symmetric $1 \mathrm{H} 2 \mathrm{D}$ instances

From Table 3 it can be concluded that FR is more efficient than BC for fast good feasible solutions of $1 \mathrm{H} 2 \mathrm{D}$ tables. In several runs (marked with $\ddagger$ ) $\mathrm{BC}$ could not find a better solution than FR within the time limit. It is worth noting that for all the 1H2D instances FR provided solutions with gaps below 6\%. For the real-world general instances of Table 4 the situation is slightly different. These instances are not guaranteed to have a hierarchical structure, and this may explain why FR is not as competitive as for $1 \mathrm{H} 2 \mathrm{D}$ tables. FR provided a better gap than $\mathrm{BC}$ within the same CPU time in 17 of the 34 instances, and both FR and BC provided the same gap in six adittional cases. In six of these cases BC could not improve the FR solution within the two hours time limit. In the remaining instances BC outperformed FR.

\subsection{Comparison between fix-and-relax with block coordinate descent and plain branch-and-cut}

As mentioned in section 3.2 since FR can provide good feasible solutions faster in average than $\mathrm{BC}, \mathrm{BCD}$ was warm started with the FR solution. This hybrid approach was named $\mathrm{FR}+\mathrm{BCD}$.

The BCD algorithm performed in all cases a loop with two clusters, each one with a half of the sensitive cells, partitioned at random. At exit, the CPU computation time and the objective function value were saved. This CPU time was added to the FR CPU time and compared to the CPU time used by BC. We also took into account whether the sensitive cells had been correctly protected in the final solutions, since accuracy errors might be present in some instances, making actually infeasible the protected table. This accuracy errors are due to the big-M constraints $z_{i}^{+} \leq u_{z_{i}} y_{i}$ and $z_{i}^{-} \leq-l_{z_{i}}\left(1-y_{i}\right)$ of (3) and (4), since $u_{z_{i}}$ and $-l_{z_{i}}$ can take very large values. 


\begin{tabular}{|c|c|c|c|c|c|c|c|}
\hline instance & $T_{F R}$ & $G A P_{F R} \%$ & $G A P_{B C} \%$ & $\Delta(B C, F R)$ & $G A P_{B C}^{u p} \%$ & $T_{B C}^{u p}$ & $\Delta\left(T_{R S}^{u p}, T_{F R}\right)$ \\
\hline australia_ABS & 6,05 & 73,87 & 3,84 & $-70,03$ & 7,40 & 2,45 & $-3,6$ \\
\hline bts4 & 6332,15 & 66,57 & 74,16 & 7,59 & $\neq$ & $\ddagger$ & $\ddagger$ \\
\hline cbs & 2,87 & 100,00 & 100,00 & 0,00 & 0,00 & 2,88 & 0,01 \\
\hline dale & 595,85 & 48,44 & 48,44 & 0,00 & 48,44 & 7199,96 & 6604,11 \\
\hline destatis & 204,32 & 19,53 & 99,97 & 80,44 & 1,80 & 706,48 & 502,16 \\
\hline hier13d4 & 6410,73 & 82,86 & 99,98 & 17,12 & \# & $\ddagger$ & $\ddagger$ \\
\hline hier13 & 747,29 & 6,88 & 4,90 & $-1,98$ & 4,90 & 159,24 & $-588,05$ \\
\hline hier13x13x13a & 542,72 & 5,22 & 5,22 & 0,00 & 4,94 & 690,15 & 147,43 \\
\hline hier13x13x13b & 584,92 & 5,89 & 5,24 & $-0,65$ & 5,24 & 369,13 & $-215,79$ \\
\hline hier $13 \times 13 \times 13 c$ & 542,86 & 5,63 & 4,99 & $-0,65$ & 4,99 & 243,43 & $-299,43$ \\
\hline hier13x13x13d & 178,12 & 4,69 & 5,27 & 0,58 & 2,40 & 340,47 & 162,35 \\
\hline hier $13 \times 13 \times 13 e$ & 336,72 & 5,39 & 4,40 & $-0,99$ & 4,40 & 269,82 & $-66,9$ \\
\hline hier $13 \times 13 \times 7 d$ & 34,72 & 5,58 & 7,19 & 1,61 & 4,96 & 69,32 & 34,6 \\
\hline hier $13 \times 7 \times 7 d$ & 2,71 & 4,82 & 12,35 & 7,53 & \$ & $\ddagger$ & $\ddagger$ \\
\hline hier16 & 4854,46 & 59,48 & 63,07 & 3,59 & $\neq$ & $\ddagger$ & $\dagger$ \\
\hline hier16x16x16a & 2803,76 & 44,96 & 48,80 & 3,84 & 44,71 & 3706,65 & 902,89 \\
\hline hier16x16x16b & 3401,2 & 33,49 & 99,95 & 66,46 & 31,89 & 6275,87 & 2874,67 \\
\hline hier16x16x16c & 3488,27 & 40,86 & 50,40 & 9,54 & 39,62 & 4926,13 & 1437,86 \\
\hline hier16x16x16d & 3776,81 & 57,66 & 63,32 & 5,66 & 57,07 & 5369,03 & 1592,22 \\
\hline hier16x16x16e & 3862,21 & 46,55 & 46,87 & 0,32 & $\ddagger$ & $\ddagger$ & $\ddagger$ \\
\hline nine $5 \mathrm{~d}$ & 6133,25 & 67,69 & 99,99 & 32,30 & $\neq$ & $\ddagger$ & $\ddagger$ \\
\hline osorio & 1,86 & 0,00 & 0,00 & 0,00 & 0,00 & 1,03 & $-0,83$ \\
\hline sbs2008_C & 543,88 & 50,11 & 3,36 & $-46,75$ & 21,77 & 32,24 & $-511,64$ \\
\hline sbs2008_E & 4,56 & 4,73 & 4,73 & 0,00 & 4,73 & 2,94 & $-1,62$ \\
\hline table1 & 0,62 & 8,38 & 13,43 & 5,06 & 4,92 & 1,25 & 0,63 \\
\hline table3 & 1909,18 & 25,39 & 15,07 & $-10,32$ & 17,11 & 408,3 & $-1500,88$ \\
\hline table4 & 1196,86 & 25,39 & 17,30 & $-8,09$ & 18,60 & 511,64 & $-685,22$ \\
\hline table5 & 720,49 & 22,80 & 20,20 & $-2,60$ & 20,25 & 216,19 & $-504,3$ \\
\hline table6 & 1,01 & 7,77 & 39,32 & 31,54 & 3,36 & 2,17 & 1,16 \\
\hline table7 & 0,1 & 1,01 & 0,41 & $-0,61$ & 0,41 & 0,02 & $-0,08$ \\
\hline table8 & 0,18 & 2,44 & 0,00 & $-2,44$ & 1,35 & 0,04 & $-0,14$ \\
\hline targus & 0,08 & 3,84 & 3,84 & 0,00 & 3,84 & 0,01 & $-0,07$ \\
\hline toy3dsarah & 25,47 & 0,34 & 7,29 & 6,94 & 0,34 & 37,46 & 11,99 \\
\hline two5in6 & 3010,89 & 66,04 & 99,99 & 33,95 & 63,91 & 7200,45 & 4189,56 \\
\hline
\end{tabular}

Table 4: Comparison between fix-an-relax and plain branch-and-cut for real instances 


\begin{tabular}{|c|c|c|c|c|}
\hline \multirow{3}{*}{\multicolumn{2}{|c|}{$\mathrm{FR}+\mathrm{BCD}<\mathrm{BC}$}} & & \multicolumn{2}{|c|}{ Objective Function } \\
\hline & & & $\mathrm{FR}+\mathrm{BCD}<\mathrm{BC}$ & $\mathrm{FR}+\mathrm{BCD}>\mathrm{BC}$ \\
\hline & & & & \\
\hline \multirow{11}{*}{ Time } & \multirow{11}{*}{$\mathrm{FR}+\mathrm{BCD}>\mathrm{BC}$} & mean (sd) $\Delta F$ & $-1.24(1.08)$ & $1.86(1.46)$ \\
\hline & & $\max |\Delta F|$ & -4.23 & 7.26 \\
\hline & & mean (sd) $\Delta T$ & $-1564(1872)$ & $-883(1281)$ \\
\hline & & $\max |\Delta T|$ & -6641 & -6351 \\
\hline & & $\mathrm{N}\left[\mathrm{N}_{\text {asym }} ; \mathrm{N}_{\text {sym }}\right]$ & $58[27 ; 31]$ & $119[93 ; 26]$ \\
\hline & & & & \\
\hline & & mean (sd) $\Delta F$ & $-1.55(1.43)$ & $0.83(1.07)$ \\
\hline & & $\max |\Delta F|$ & -3.89 & 4.17 \\
\hline & & mean (sd) $\Delta T$ & $146(207)$ & $49(38)$ \\
\hline & & $\max |\Delta T|$ & 508 & 182 \\
\hline & & $\mathrm{N}\left[\mathrm{N}_{\text {asym }} ; \mathrm{N}_{\text {sym }}\right]$ & $5[4 ; 1]$ & $58[56 ; 2]$ \\
\hline
\end{tabular}

Table 5: Summary of results for 1H2D instances, in the comparison between FR+BCD versus BC.

With regard to $1 \mathrm{H} 2 \mathrm{D}$ instances, it has been observed that the extra time needed by the BCD stage is related to the number of sensitive cells, although with considerable variability especially if the table is large. The 16 tables with more than 50,000 sensitive cells consumed between 114 and 492 seconds, with a median time of 255 seconds. In 104 instances with less than 10,000 sensitive cells the median time was 29.7 seconds. Compared to the time employed by the FR stage, it took about $40 \%$ of that time (median proportion): in 18 instances out of $240 \mathrm{BCD}$ lasted longer than FR, generally in tables with high density of sensitive cells.

Sixty-one tables improved the objective function after the BCD stage, and the others remained in the same value (not necessarily in the same solution). The median change in the objective function with respect to the value attained by FR was 3\%, with a maximum of $10 \%$. Improving the solution requires also more time: $54.6 \%$ of FR time, instead of $37 \%$ for the tables not improved. We observed a higher rate of success among the tables with high density of sensitive cells: an odd of 33 versus 47 for tables with $30 \%$ of sensitive cells, compared to 28 versus 132 for tables with $15 \%$ or lower proportion. The table size or the asymmetry degree in the protection levels were not related to improvement in the objective function.

Table 5]summarizes the results with 1H2D instances for two factors: solution times (in rows) and objective function values (in columns) between BC and FR+BCD. The two categories for each factor are either FR+BCD outperfomed $\mathrm{BC}$ ("FR+BCD $<\mathrm{BC}$ ", i.e., less CPU time or a lower objective function value) or the opposite ("FR+BCD $>$ BC"). Each of the four cells shows the number of instances ("N"), and some statistics (mean, standard deviation, maximum) about the change in both factors: $\Delta_{F}$ is the percentage change in the objective function, $\Delta_{T}$ is the absolute change in the time. Comparing left versus right columns, we can see small differences between the percentage changes in objective function values (they range from $-4.23 \%$ to $7.26 \%$ ). However, comparing above versus below rows, we can see large differences with respect to solution times: 1564 and 883 seconds in favor of FR+BCD (177 cases) against 146 and 49 seconds (63 cases) in favor of BC.

For the real-world tables, FR+BCD got better solutions than FR in 18 instances after a BCD cycle, whereas it did not improve the FR objective function in 16 cases. Table 6 reports the results obtained. Columns " $F$ " and " $T$." provide, respectively, the objective function and CPU solution times for each method, BC, FR and FR+BCD or BCD. Column " $\triangle\left(F_{F R}, F_{F R+B C D}\right)$ " provides the relative change (as a percentage) in the objective function between the FR and FR+BCD solutions. The rows are ordered by $\Delta\left(F_{F R}, F_{F R+B C D}\right)$; the first instance shows a negative change 


\begin{tabular}{|c|c|c|c|c|c|c|c|}
\hline instance & $F_{B C}$ & $F_{F R}$ & $F_{F R+B C D}$ & $T_{B C}$ & $T_{F R}$. & $T_{B C D}$ & $\Delta\left(F_{F R}, F_{F R+B C D}\right)$ \\
\hline table6: & 28331962414 & 29686800000 & 29899600000 & 2.17 & 1 & 0.38 & -0.72 \\
\hline dale & 256 & 256 & 256 & 7199.96 & 595.9 & 596.62 & 0 \\
\hline hier13 & 434834824.5 & 444063000 & 444063000 & 1312.7 & 747.3 & 8.18 & 0 \\
\hline hier13d4 & $5.11488 \mathrm{e}+12$ & 6143970000 & 6143970000 & 7201.23 & 6410.7 & 2769.53 & 0 \\
\hline hier13x13x13a & 434834824.5 & 436127000 & 436127000 & 895.44 & 542.7 & 6.16 & 0 \\
\hline hier $13 \times 13 \times 13 b$ & 44385.67 & 44865.8 & 44865.8 & 1444.94 & 584.9 & 7.14 & 0 \\
\hline hier $13 \times 13 \times 13 c$ & 368036.2 & 370564 & 370564 & 1561.69 & 542.9 & 8.47 & 0 \\
\hline hier13x13x13d & 414115.44 & 424074 & 424074 & 340.48 & 178.1 & 6.89 & 0 \\
\hline hier13x13x13e & 4644973.87 & 4693570 & 4693570 & 269.83 & 336.7 & 6.83 & 0 \\
\hline hier13x7x7d & 594401 & 593370 & 593370 & 29.19 & 2.7 & 0.24 & 0 \\
\hline hier16 & 591756145.8 & 556221000 & 556221000 & 7200.41 & 4854.5 & 130.81 & 0 \\
\hline hier16x16x16b & 74891.53 & 76700.4 & 76700.4 & 7200.44 & 3401.2 & 121.52 & 0 \\
\hline osorio & 13 & 13 & 13 & 1.8 & 1.9 & 1.35 & 0 \\
\hline sbs2008_E & 109959.57 & 109960 & 109960 & 2.95 & 4.6 & 0.13 & 0 \\
\hline table7 & 9970266227 & 10031200000 & 10031200000 & 0.04 & 0.1 & 0.1 & 0 \\
\hline toy3dsarah & $5.0747 e+14$ & $5.07506 e+14$ & $5.07506 \mathrm{e}+14$ & 37.46 & 25.5 & 0.22 & 0 \\
\hline hier $13 \times 13 \times 7 d$ & 1684140 & 1695250 & 1686430 & 241.81 & 34.7 & 2.59 & 0.52 \\
\hline table8 & 439 & 450 & 445 & 0.09 & 0.2 & 0.18 & 1.11 \\
\hline hier16x16x16a & 529703489.9 & 532145000 & 525466000 & 7200.31 & 2803.8 & 372.61 & 1.26 \\
\hline targus & 1103759.75 & 1103760 & 1088480 & 0.02 & 0.1 & 0.08 & 1.38 \\
\hline nine $5 \mathrm{~d}$ & $6.20788 \mathrm{e}+12$ & 1215790000 & 1191810000 & 7200.51 & 6133.3 & 2389.56 & 1.97 \\
\hline table5 & 10154665.5 & 11094800 & 10837600 & 7200.21 & 720.5 & 258.66 & 2.32 \\
\hline hier16x16x16c & 604844.28 & 620633 & 601548 & 7200.39 & 3488.3 & 672.13 & 3.08 \\
\hline table4 & 10290147784 & 11843300000 & 11433900000 & 7200.27 & 1196.9 & 142.92 & 3.46 \\
\hline hier16x16x16e & 9543201.06 & 9485820 & 9077750 & 7200.41 & 3862.2 & 692.34 & 4.3 \\
\hline table1 & $2.93185 e+13$ & $3.04227 \mathrm{e}+13$ & $2.89576 \mathrm{e}+13$ & 1.45 & 0.6 & 0.54 & 4.82 \\
\hline two5in6 & 707133564.8 & 751514000 & 713214000 & 7200.45 & 3010.9 & 169.86 & 5.1 \\
\hline hier16x16x16d & 752648610.3 & 765577000 & 725869000 & 7200.31 & 3776.8 & 789.54 & 5.19 \\
\hline table3 & $1.20849 \mathrm{e}+12$ & $1.39866 \mathrm{e}+12$ & $1.29248 \mathrm{e}+12$ & 7200.24 & 1909.2 & 171.17 & 7.59 \\
\hline destatis & 234541294 & 286199000 & 241329000 & 2528.91 & 204.3 & 29.83 & 15.68 \\
\hline bts4 & 4114851966 & 3180710000 & 2592590000 & 7200.6 & 6332.2 & 6002.29 & 18.49 \\
\hline sbs2008_C & 320835.46 & 621448 & 459655 & 66.34 & 543.9 & 0.43 & 26.03 \\
\hline australia_ABS & 651 & 2396 & 746 & 2.91 & 6.05 & 4.46 & 68.86 \\
\hline $\operatorname{cbs} \dagger$ & 0 & 268 & 0 & 2.88 & 2.9 & 1.79 & 100 \\
\hline
\end{tabular}

$\dagger$ cbs instance has a global optimum of zero because all the sensitive cells have null weights in the objective function.

Table 6: Comparison between plain branch-and-cut and FR+BCD with real-world instances.

because the solution reached by FR was actually not feasible, due to slight deviations in some sensitive cells beyond their protection levels, but undetectable with the (already tight) infeasibility tolerance in use by the solver (cf. big-M issue discussed above). In general, FR already provided a good solution for instances which could not be improved by BCD; this FR solution was close to the one obtained by $\mathrm{BC}$, but it was computed faster. On the other hand, it is remarkable that most of the instances where the BCD cycle could improve the solution were difficult for the $\mathrm{BC}$ scheme, which used to exhaust the time limit.

Table 7 summarizes the results for the real-world instances with respect to CPU time and objective function values. The structure of this table is similar to that of Table 5, but with an additional central column. This central column corresponds to instances without relevant differences in the objective function value (i.e., $\left(F_{F R+B C D}-F_{B C}\right) / F_{B C}$ less than $\left.5 \%\right)$. Each cell of Table 7 reports the number and names of its instances. The first row includes the instances that were solved faster with FR+BCD than with $\mathrm{BC}$, and the instances that could not be solved in the 2-hour time limit by BC, but they could by FR+BCD. Moreover, some instances were not suitably protected: bts4, dale, table1, table3, table4, table5, table6 and table7 present some sensitive cells unprotected in the BC solution; dale, table5 and table6 had the same problem with FR, and $\mathrm{BCD}$ was in trouble as well with bts4 and table6: in general, $\mathrm{FR}+\mathrm{BCD}$ dealt better than $\mathrm{BC}$ with these difficult instances. 


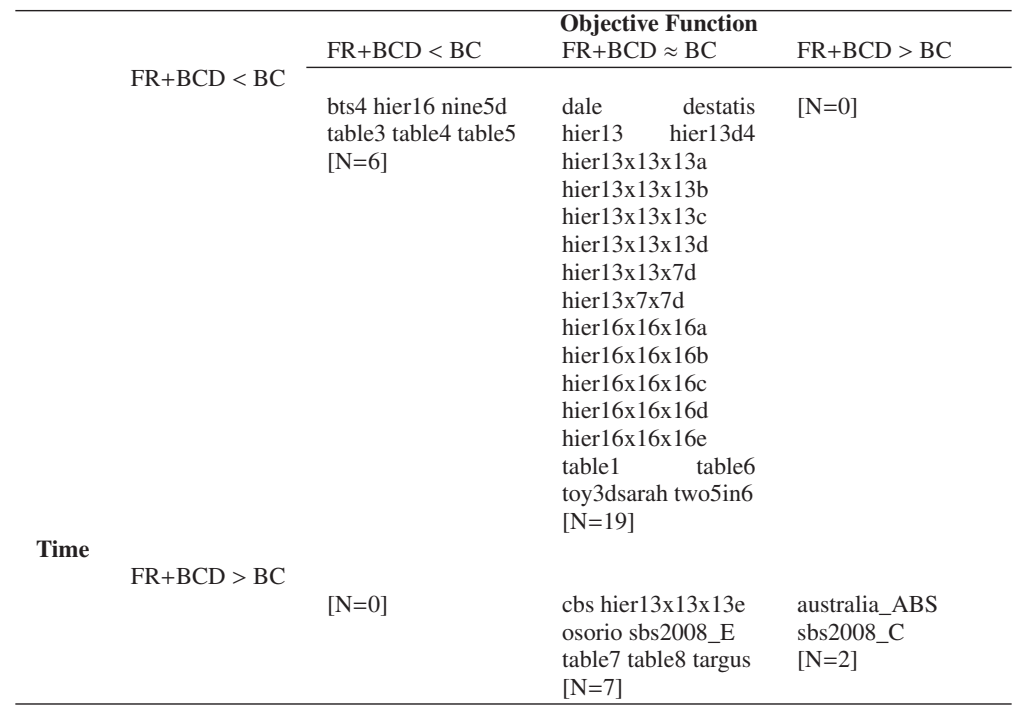

Table 7: Summary of results for real instances, in the comparison between FR+BCD versus BC.

Nine tables were solved faster with the pure BC scheme, but it is worth noting that only two (sbs2008_C and hier13x13x13e) can be considered as challenging, since they needed more than one minute to be solved, whereas four (osorio, table7, table8 and targus) have few sensitive cells and could be solved very quickly by both $\mathrm{FR}+\mathrm{BCD}$ and $\mathrm{BC}$.

To sum up, Table 7 shows that the combination FR+BCD is competitive with $\mathrm{BC}$ in the solution's quality, and, in addition, it protects the table in significantly less time.

\subsection{Comparison between fix-and-relax and other heuristics}

Current state-of-the-art MILP solvers can be turned into heuristic approaches by tuning some of their pre-build heuristics. For a fair comparison, FR is tested in this section against feasibility pump (FP), relaxation induced neighborhod search (RINS), and FR+BC (warm starting CPLEX from the FR solution) with and without polishing.

\subsubsection{Fix-and-relax and feasibility pump heuristics}

FP [17] is considered an efficient heuristic for the fast computation of hopefully good initial feasible solutions to MILPs. We used the objective feasibility pump (oFP) [1], which is more efficient than FP in terms of quality of the solution and the analytic center feasibility pump (ACFP) [2], which was introduced as a good alternative in some MILP instances (either in time or quality of the solution). Table 8 shows a comparison between FR and these FP variants for real instances. It reports the primal gap of the FR and FP solutions (columns " $G A P_{F R} \%$ " and "GAP $F P \%$ ", respectively), the CPU time required by FR and FP to compute the feasible solution (columns " $T_{F R}$ " and " $T_{F P}$ ", respectively), and the difference between both methods in CPU times and gaps (columns " $\triangle\left(T_{F P}, T_{F R}\right)$ " and " $\triangle(F P, F R)$ ", respectively). We ran both oFP and AC-FP. Table 8 only shows the result of the best FP variant, i.e., the one that provides the lowest gap, and in case of equal gaps, the fastest one. The best FP variant is clearly marked in the table. 


\begin{tabular}{|c|c|c|c|c|c|c|}
\hline instance & $G A P_{F R} \%$ & $T_{F R}$ & $G A P_{F P} \%$ & $T_{F P}$ & $\Delta\left(T_{F P}, T_{F R}\right)$ & $\Delta(F P, F R)$ \\
\hline australia_ABS & 73,87 & 6,05 & $95,90^{A C-F P}$ & 26 & 19,95 & 22,03 \\
\hline bts4 & 66,57 & 6332,15 & $74,09^{o F P}$ & 552 & $-5780,15$ & 7,52 \\
\hline cbs & 100,00 & 2,87 & $100,00^{o F P}$ & 20 & 17,13 & 0,00 \\
\hline dale & 48,44 & 595,85 & $98,52^{o F P}$ & 27 & $-568,85$ & 50,08 \\
\hline destatis & 19,53 & 204,32 & $21,93^{A C-F P}$ & 222 & 17,68 & 2,40 \\
\hline hier13d4 & 82,86 & 6410,73 & $\dagger$ & $\dagger$ & $\dagger$ & $\dagger$ \\
\hline hier13 & 6,88 & 747,29 & $58,96^{o F P}$ & 126 & $-621,29$ & 52,08 \\
\hline hier13x13x13a & 5,22 & 542,72 & $63,36^{A C-F P}$ & 122 & $-420,72$ & 58,14 \\
\hline hier13x13x13b & 5,89 & 584,92 & $53,36^{A C-F P}$ & 235 & $-349,92$ & 47,47 \\
\hline hier $13 \times 13 \times 13 c$ & 5,63 & 542,86 & $54,01^{\text {oFP }}$ & 217 & $-325,86$ & 48,38 \\
\hline hier13x13x13d & 4,69 & 178,12 & $99,86^{o F P}$ & 132 & $-46,12$ & 95,17 \\
\hline hier13x13x13e & 5,39 & 336,72 & $99,87^{o F P}$ & 128 & $-208,72$ & 94,48 \\
\hline hier $13 \times 13 \times 7 d$ & 5,58 & 34,72 & $60,49^{A C-F P}$ & 13 & $-21,72$ & 54,91 \\
\hline hier13x7x7d & 4,82 & 2,71 & $73,56^{o F P}$ & 2 & $-0,71$ & 68,73 \\
\hline hier16 & 59,48 & 4854,46 & $68,36^{A C-F P}$ & 2852 & $-2002,46$ & 8,89 \\
\hline hier16x16x16a & 44,96 & 2803,76 & $99,99^{o F P}$ & 4537 & 1733,24 & 55,03 \\
\hline hier16x16x16b & 33,49 & 3401,2 & $99,91^{\text {oFP }}$ & 3742 & 340,8 & 66,42 \\
\hline hier16x16x16c & 40,86 & 3488,27 & $99,93^{\text {oFP }}$ & 3937 & 448,73 & 59,07 \\
\hline hier16x16x16d & 57,66 & 3776,81 & $66,88^{A C-F P}$ & 2706 & $-1070,81$ & 9,22 \\
\hline hier16x16x16e & 46,55 & 3862,21 & $81,19^{o F P}$ & 4430 & 567,79 & 34,64 \\
\hline nine $5 d$ & 67,69 & 6133,25 & $\dagger$ & $\dagger$ & $\dagger$ & $\dagger$ \\
\hline osorio & 0,00 & 1,86 & $27,65^{o F P}$ & 0 & $-1,86$ & 27,65 \\
\hline sbs2008_C & 50,11 & 543,88 & $82,64^{o F P}$ & 12 & $-531,88$ & 32,53 \\
\hline sbs2008_E & 4,73 & 4,56 & $74,15^{\text {oFP }}$ & 2 & $-2,56$ & 69,42 \\
\hline table1 & 8,38 & 0,62 & $2,17^{o F P}$ & 0 & $-0,62$ & $-6,20$ \\
\hline table3 & 25,39 & 1909,18 & $100,00^{o F P}$ & 323 & $-1586,18$ & 74,61 \\
\hline table4 & 25,39 & 1196,86 & $96,81^{A C-F P}$ & 379 & $-817,86$ & 71,42 \\
\hline table6 & 7,77 & 1,01 & $9,05^{o F P}$ & 0 & $-1,01$ & 1,28 \\
\hline table7 & 1,01 & 0,1 & $69,21^{o F P}$ & 0 & $-0,1$ & 68,20 \\
\hline table8 & 2,44 & 0,18 & $6,51^{o F P}$ & 0 & $-0,18$ & 4,07 \\
\hline targus & 3,84 & 0,08 & $0,92^{\text {oFP }}$ & 0 & $-0,08$ & $-2,92$ \\
\hline toy3dsarah & 0,34 & 25,47 & $65,07^{\text {oFP }}$ & 5 & $-20,47$ & 64,73 \\
\hline two5in6 & 66,04 & 3010,89 & $61,91^{A C-F P}$ & 5234 & 2223,11 & $-4,13$ \\
\hline
\end{tabular}

Table 8: Comparison between fix-and-relax and feasibility pump for real instances.

It is clearly seen that FR outperformed FP for CTA in terms of quality of the solution. In most cases, FR provided a better gap than FP by a big difference. Only in three instances FP was better. FP reached the time limit without a feasible solution in two instances. However, FP is in general faster than FR in order to find a feasible solution. It can be concluded that, for the CTA problem, FR instead of FP should be used for finding good feasible solutions within a reasonable short time.

\subsubsection{Fix-and-relax and RINS and local branching heuristics}

RINS [12] is a heuristic that explores a neighborhood of the current incumbent solution and the continuous relaxation at a node $h$ of the BC tree to try to find a new and improved incumbent. CPLEX BC incorporates RINS, allowing the user to control how often to apply the heuristic through a frequency parameter $f$. A value $f>0$ means that RINS is applied at nodes $h=0, f, 2 f, \ldots$ while for $f=0$ CPLEX automatically decides when to apply the heuristic. The results of Table 4 were obtained with $f=0$; as it was shown in that table, FR outperformed BC in a considerable percentage of real-world instances. Table 9 adds a comparison between FR and BC with $f=50$. The meaning of columns is the same as in Table 4 The value of $f$, either 0 or 50 , is reported in the new column $\operatorname{RINS}_{f}$. We only considered the subset of real-world 


\begin{tabular}{|c|c|c|c|c|c|c|c|c|}
\hline instance & $T_{F R}$ & $G A P_{F R} \%$ & $\operatorname{RINS}_{f}$ & $G A P_{B C} \%$ & $\Delta(B C, F R)$ & $G A P_{B C}^{u p} \%$ & $T_{B C}^{u p}$ & $\Delta\left(T_{F R}, T_{B C}^{u p}\right)$ \\
\hline \multirow[t]{2}{*}{ bts4 } & \multirow{2}{*}{6332,15} & \multirow[b]{2}{*}{66,57} & 0 & 74,16 & 7,59 & 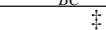 & 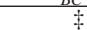 & \$ \\
\hline & & & 50 & 100 & 33,43 & $\ddagger$ & $\ddagger$ &  \\
\hline \multirow[t]{2}{*}{ dale } & \multirow{2}{*}{595,85} & \multirow{2}{*}{48,44} & 0 & 48,44 & 0 & 48,44 & 7199,96 & 6604,11 \\
\hline & & & 50 & 48,44 & 0 & $\ddagger$ & $\ddagger$ & $\neq$ \\
\hline \multirow[t]{2}{*}{ destatis } & \multirow{2}{*}{204,32} & \multirow{2}{*}{19,53} & 0 & 99,97 & 80,44 & 1,8 & 706,48 & 502,16 \\
\hline & & & 50 & 99,97 & 80,44 & 1,78 & 3090,16 & 2885,84 \\
\hline \multirow[t]{2}{*}{ hier13 } & \multirow{2}{*}{747,29} & \multirow{2}{*}{6,88} & 0 & 4,9 & $-1,98$ & 4,9 & 159,24 & $-588,05$ \\
\hline & & & 50 & 99,98 & 93,1 & 4,9 & 1239,3 & 492,01 \\
\hline \multirow[t]{2}{*}{ hier13x13x13a } & \multirow{2}{*}{542,72} & \multirow{2}{*}{5,22} & 0 & 5,22 & 0 & 4,94 & 690,15 & 147,43 \\
\hline & & & 50 & 99,99 & 94,77 & 4,94 & 1426,84 & 884,12 \\
\hline \multirow{2}{*}{ hier $13 \times 13 \times 13 b$} & \multirow{2}{*}{584,92} & \multirow{2}{*}{5,89} & 0 & 5,24 & $-0,65$ & 5,24 & 369,13 & $-215,79$ \\
\hline & & & 50 & 99,98 & 94,09 & 4,87 & 2742,67 & 2157,75 \\
\hline \multirow{2}{*}{ hier $13 \times 13 \times 13 c$} & \multirow{2}{*}{542,86} & \multirow{2}{*}{5,63} & 0 & 4,99 & $-0,65$ & 4,99 & 243,43 & $-299,43$ \\
\hline & & & 50 & 99,98 & 94,34 & 4,99 & 3405,16 & 2862,3 \\
\hline \multirow[t]{2}{*}{ hier $13 \times 13 \times 7 d$} & \multirow{2}{*}{34,72} & \multirow{2}{*}{5,58} & 0 & 7,19 & 1,61 & 4,96 & 69,32 & 34,6 \\
\hline & & & 50 & 38,93 & 33,34 & 4,84 & 158,96 & 124,24 \\
\hline \multirow[t]{2}{*}{ hier $13 \times 7 \times 7 d$} & \multirow{2}{*}{2,71} & \multirow{2}{*}{4,82} & 0 & 12,35 & 7,53 & $\ddagger$ & $\ddagger$ & 末 \\
\hline & & & 50 & 24,1 & 19,28 & 4,53 & 20,09 & 17,38 \\
\hline \multirow[t]{2}{*}{ hier16 } & $1854-4$ & $50-18$ & 0 & 63,07 & 3,59 & $\ddagger$ & $\ddagger$ & $\$$ \\
\hline & 4854,46 & 59,48 & 50 & 100 & 40,52 & $\ddagger$ & $\ddagger$ & + \\
\hline hier16x16x16a & 280376 & 4406 & 0 & 48,8 & 3,84 & 44,71 & 3706,65 & 902,89 \\
\hline & 2800,10 & 44,90 & 50 & 100 & 55,03 & 43,87 & 7200,57 & 4396,81 \\
\hline hier16x $16 \times 16 \mathrm{~d}$ & 377681 & 5766 & 0 & 63,32 & 5,66 & 57,07 & 5369,03 & 1592,22 \\
\hline & & & 50 & 100 & 42,33 & 56,08 & 7200,59 & 3423,78 \\
\hline hier16x16x16e & 386221 & 4655 & 0 & 46,87 & 0,32 & $\ddagger$ & \$ & * \\
\hline & & & 50 & 99,96 & 53,41 & 46,18 & 7200,52 & 3338,31 \\
\hline table3 & 100918 & 2539 & 0 & 15,07 & $-10,32$ & 17,11 & 408,3 & $-1500,88$ \\
\hline & 1909,18 & 25,39 & 50 & 100 & 74,61 & 16,78 & 2303,34 & 394,16 \\
\hline table4 & 119686 & 2539 & 0 & 17,3 & $-8,09$ & 18,6 & 511,64 & $-685,22$ \\
\hline & 1196,86 & 25,39 & 50 & 100 & 74,61 & 14,64 & 2163,97 & 967,11 \\
\hline table5 & 720.49 & 228 & 0 & 20,2 & $-2,6$ & 20,25 & 216,19 & $-504,3$ \\
\hline & 720,49 & 22,8 & 50 & 100 & 77,2 & 16,96 & 1441,96 & 721,47 \\
\hline
\end{tabular}

Table 9: Comparison between FR and BC with frequency RINS $f$ equal to 0 and 50 for some real-world instances

instances whose BC tree had more than 50 nodes. From Table 9 it can be concluded that FR still outperforms BC with the RINS heuristic using $f=50$.

We additionally tried RINS frequencies $f \in\{100,150,200\}$, obtaining exactly the same results (they are thus omitted in Table 9). As stated above, CPLEX always applies the RINS heuristic at node 0 for any $f>0$. We noted that, since RINS is an expensive heuristic, it exhausted most of the allowed time (that of the FR heuristic) at node 0, making irrelevant the particular value of $f$. Therefore, at least for this particular application, RINS $f=0$ seems to be the best choice. Indeed, we noted that when $f=0$ CPLEX does not apply RINS to node 0 in many instances.

The local branching ( $\mathrm{LBr}$ ) heuristic also explores the neighborhood of an incumbent solution, but by adding constraints based on the number of binary variables flipping their values with respect the incumbent [18]. Running CPLEX with the LBr heuristic, and setting as time limit the CPU time of FR, we only observed differences with RINS $f=0$ for five instances of Table 9. hier13x13x7d (solutions of $6.2 \%$ and $7.2 \%$ gaps for LBr and RINS, respectively), hier13x7x7d (24.1\% gap for LBr, 12,4\% gap for RINS), hier16 (61.2\% for $\mathrm{LBr}, 63.0 \%$ for RINS), hier16x16x16a (44.4\% for LBr, $49.0 \%$ for RINS), and hier16x16x16d (62.4\% for $\mathrm{LBr}$, $63,0 \%$ for RINS). LBr only clearly outperformed RINS $f=0$ in hier16x16x16a; for that instance, $\mathrm{LBr}$ was also more efficient than FR. 


\begin{tabular}{|c|c|c|c|c|c|c|c|c|}
\hline instance & $T_{F R}$ & $G A P_{F R} \%$ & $T_{F R+B C}$ & $G A P_{F R+B C} \%$ & $T_{B C}$ & $G A P_{B C} \%$ & $\Delta(F R+B C, B C)$ & $\Delta\left(T_{F R+B C}, T_{B C}\right)$ \\
\hline asym-40-50-5 & 72.76 & 1.89 & 264.45 & 0.47 & 306.46 & 0.35 & 0.12 & -42.01 \\
\hline asym-40-50-15 & 158.75 & 3.13 & $\dagger^{(1223.55,1)}$ & 0.71 & $\dagger^{(1357.15,3)}$ & 0.67 & 0.04 & -133.60 \\
\hline asym-40-50-30 & 210.51 & 5.41 & $\dagger(1606.53,2)$ & 0.82 & $\dagger(2062.43,3)$ & 1.04 & -0.23 & -455.90 \\
\hline asym-40-60-5 & 95.40 & 1.75 & 259.40 & 0.40 & 231.36 & 0.40 & 0.00 & 28.04 \\
\hline asym-40-60-15 & 193.37 & 3.11 & $\dagger^{(1352.60,2)}$ & 0.93 & $\dagger^{(1430.58,4)}$ & 1.02 & -0.09 & -77.98 \\
\hline asym-40-60-30 & 314.96 & 5.07 & $\dagger^{(2181.63,6)}$ & 1.40 & $\dagger^{(2040.04,6)}$ & 1.21 & 0.19 & 141.59 \\
\hline asym-50-50-5 & 110.54 & 1.53 & $\dagger(560.24,1)$ & 0.43 & 476.49 & 0.35 & 0.08 & 83.75 \\
\hline asym-50-50-15 & 186.95 & 2.86 & $\dagger^{(1403.85,3)}$ & 0.90 & $\dagger^{(1533.78,4)}$ & 0.93 & -0.03 & -129.93 \\
\hline asym-50-50-30 & 333.50 & 5.81 & $\dagger^{(2417.91,5)}$ & 1.52 & $\dagger^{(2284.80,5)}$ & 1.74 & -0.22 & 133.11 \\
\hline asym-50-60-5 & 153.14 & 1.06 & 268.43 & 0.64 & 286.51 & 0.48 & 0.16 & -18.08 \\
\hline asym-50-60-15 & 278.72 & 2.73 & $\dagger^{(1263.52,3)}$ & 1.24 & $\dagger^{(1408.72,3)}$ & $\ddagger^{(8.67,2)}$ & -7.43 & -145.21 \\
\hline asym-50-60-30 & 416.11 & 5.54 & $\dagger^{(2768.88,8)}$ & 1.60 & $\dagger(2665.53,7)$ & $\ddagger^{(1.74,1)}$ & -0.14 & 103.35 \\
\hline sym-40-50-5 & 8.99 & 2.00 & 41.51 & 0.40 & 29.10 & 0.68 & -0.28 & 12.40 \\
\hline sym-40-50-15 & 115.34 & 4.21 & 1114.50 & 0.75 & 720.22 & 0.80 & -0.06 & 394.29 \\
\hline sym-40-50-30 & 371.35 & 4.62 & $\dagger^{(3097.98,4)}$ & 1.93 & $\dagger^{(3131.25,3)}$ & 1.77 & 0.16 & -33.27 \\
\hline sym-40-60-5 & 10.52 & 2.68 & 32.82 & 0.63 & 60.05 & 0.52 & 0.11 & -27.23 \\
\hline sym-40-60-15 & 102.29 & 2.16 & 1731.73 & 0.86 & 1381.75 & 0.82 & 0.04 & 349.98 \\
\hline sym-40-60-30 & 800.45 & 4.40 & $\dagger^{(3600,5)}$ & 3.22 & $\dagger(3600,5)$ & 6.55 & -3.33 & 0 \\
\hline sym-50-50-5 & 25.47 & 1.88 & 103.45 & 0.50 & 85.12 & 0.57 & -0.07 & 18.33 \\
\hline sym-50-50-15 & 166.33 & 3.66 & $\dagger^{(2370.98,1)}$ & 0.97 & $\dagger^{(1728.63,1)}$ & 0.75 & 0.22 & 642.35 \\
\hline sym-50-50-30 & 511.19 & 3.45 & $\dagger^{(3600,5)}$ & 2.54 & $\dagger^{(3600,5)}$ & 4.46 & -1.92 & 0 \\
\hline sym-50-60-5 & 56.80 & 1.59 & 80.64 & 0.54 & 134.79 & 0.36 & 0.17 & -54.15 \\
\hline sym-50-60-15 & 279.63 & 2.46 & 2347.58 & 0.91 & 1894.95 & 0.74 & 0.17 & 452.63 \\
\hline sym-50-60-30 & 833.45 & 3.93 & $\dagger^{(3600,5)}$ & 3.54 & $\dagger^{(3600,5)}$ & 6.31 & -2.77 & 0 \\
\hline
\end{tabular}

Table 10: Using the fix-and-relax solution to warm start CPLEX branch-and-cut

\subsection{Using fix-and-relax to warm start branch-and-cut}

Table 10 shows the results obtained with FR+BC (i.e., warm starting BC with the FR solution) on $1 \mathrm{H} 2 \mathrm{D}$ tables. The table reports the CPU computation time and gap (as a percentage) of the FR solution (columns " $T_{F R}$ " and "GAP $F R$ \%"). The same information is provided for the FR+BC solution using a $1 \%$ optimality gap (columns " $T_{F R+B C}$ " and $G A P_{F R+B C} \%$ ); and for CPLEX BC without starting point with the same $1 \%$ optimality gap (columns $T_{B C}$ and $G A P_{B C} \%$ ). Columns $\triangle(F R+B C, B C)$ and $\triangle\left(T_{F R+B C}, T_{B C}\right)$ give the difference in gap and CPU time between the $\mathrm{FR}+\mathrm{BC}$ and $\mathrm{BC}$ solutions. A time limit of one hour was considered for these runs. Some $\mathrm{FR}+\mathrm{BC}$ or $\mathrm{BC}$ executions were unable to find a solution of $1 \%$ optimality gap within this time limit; these are clearly marked in Table 10 BC could not find a feasible solution within the time limit for three instances, which are also clearly marked in the table. In those situations the average gap reported in Table 10 may be greater than $1 \%$.

$\mathrm{FR}+\mathrm{BC}$ provided a lower gap than $\mathrm{BC}$ in 12 of 24 cases. In addition, in six of these 12 cases the $\mathrm{CPU}$ time of $\mathrm{FR}+\mathrm{BC}$ was inferior. These six successful FR+BC executions are marked in boldface in Table 10. These results are not entirely satisfactory, since it could be expected that providing a good incumbent from the beginning would significantly reduce the computational burden for all the instances, by pruning portions of the search space. In fact, we found reported similar experiences. In http://www2.isye.gatech.edu/ rcarvajal3/2012/2012-12-24_effect-of-informati the author presents an experiment with instances from MIPLIB 2010 [28] where providing the optimal solution as a warm start can actually be harmful for the performance of the solver.

We also applied the CPLEX polishing heuristic to the FR starting point. This heuristic, which can be very time consuming, tries to exploit an initial feasible solution provided to BC by 
solving an alternative branch-and-cut. We ran $\mathrm{FR}+\mathrm{BC}$ with and withoug polishing. Activating the polishing the gap was improved in $92 \%$ of the executions; however the average gap reduction was $0.4 \%$. On the other hand, in $83 \%$ of the executions the polishing significantly increased the CPU time: an average increment of 59\%. In the remaining $17 \%$ of executions the CPU time was reduced, in average, a $18 \%$. From these figures, it can be concluded that the polishing is in general very time consuming for CTA, and it is not worth the gap reduction provided.

\section{Conclusions}

FR, either alone or in combination with other heuristics such as BCD, has shown to be an efficient approach for the difficult MILP CTA problem. Initially developed for scheduling problems that can be partitioned into stages, FR has also been successfully applied to a class of hierarchical tables named 1H2D. For these tables, it was competitive against BC, and FP or RINS heuristics. For general real-world tables, FR and FR+BCD outperformed BC in $73 \%$ of the instances tested. Promising results were also obtained in a reduced set of instances by warm starting $\mathrm{BC}$ with the FR solution.

Quick tools to provide fast solutions to CTA are a necessity because of the increasing ability of NSAs to create more complex and huge tables from collected data. FR is thus an step in this direction. Combining FR with other heuristics, or embedding FR in exact approaches, like Benders reformulation, is part of the further work to be done in this field.

\section{Acknowledgments}

This work has been supported by grant MTM2012-31440 of the Spanish Government. We thank two anonymous reviewers for their comments which greatly improved the presentation of the paper.

\section{References}

[1] T. Achterberg, T. Berthold, Improving the feasibility pump, Discrete Optimization 4, 77-86, (2007).

[2] D. Baena, J. Castro, Using the analytic center in the feasibility pump, Operations Research Letters, 39, 310-317 (2011).

[3] J. Castro, Minimum-distance controlled perturbation methods for large-scale tabular data protection, European Journal of Operational Research, 171, 39-52 (2006).

[4] J. Castro, A shortest-paths heuristic for statistical data protection in positive tables, INFORMS Journal on Computing, 19(4), 520-533 (2007).

[5] J. Castro, Recent advances in optimization techniques for statistical tabular data protection, European Journal of Operational Research, 21, 257-269 (2012).

[6] J. Castro, On assessing the disclosure risk of controlled adjustment methods for statistical tabular data, International Journal of Uncertainty, Fuzziness and Knowledge-Based Systems, 20, 921-941 (2012) .

[7] J. Castro, D. Baena, Using a mathematical programming modeling language for optimal CTA, Lecture Notes in Computer Science, 5262, 1-12 (2008).

[8] J. Castro, A. Frangioni, C. Gentile, Perspective reformulations of the CTA problem with $L_{2}$ distances, Operations Research, 62(4), 891-909 (2014).

[9] J. Castro, S. Giessing, Testing variants of minimum distance controlled tabular adjustment. In: Monographs of Official Statistics, Eurostat-Office for Official Publications of the European Communities, Luxembourg, 333-343 (2006).

[10] J. Castro, J.A. González, Assessing the information loss of controlled adjustment methods in two-way tables, Lecture Notes in Computer Science, 8744, in press (2014).

[11] R.A. Dandekar, L.H. Cox, Synthetic tabular data: an alternative to complementary cell suppression, manuscript, Energy Information Administration, U.S. Department of Energy (2002). 
[12] E. Danna, E. Rothberg, C. Le Pape, Exploring relaxation induced neighborhoods to improve MIP solutions, Mathematical Programming, 102, 71-90 (2005).

[13] C. Dillenberger, L.F. Escudero, A. Wollensak, W. Zhang. On practical resource allocation for production planning and scheduling with period overlapping setups, European Journal of Operational Research, 75, 275-286 (1994).

[14] E.D. Dolan, J.J. Moré, Benchmarking optimization software with performance profiles, Mathematical Programming, A, 91, 201-13 (2002).

[15] L.F. Escudero, J. Salmerón, On a fix-and-relax framework for a class of project scheduling problems, Annals of operations research, 140, 163-188 (2005).

[16] D. Ferreira, R. Morabito, S. Rangel, Relax and fix heuristics to solve one-stage one-machine lot-scheduling models for small-scale soft drink plants, Computers \& Operations Research, 37, 684-691 (2010)

[17] M. Fischetti, F. Glover, A. Lodi, The Feasibility Pump, Mathematical Programming, 104, 91-104 (2005).

[18] M. Fischetti, A. Lodi, Local branching, Mathematical Programming, 98, 23-47 (2003).

[19] M. Fischetti, J.J. Salazar, Solving the cell suppression problem on tabular data with linear constraints, Management Science, 47, 1008-1026 (2001)

[20] S. Giessing, Pre-tabular perturbation with controlled tabular adjustment: some considerations, Lecture Notes in Computer Science, 8744, 48-61 (2014).

[21] S. Giessing, J. Höhne, Eliminating small cells from census counts tables: some considerations on transition probabilities, Lecture Notes in Computer Science, 6344, 52-65 (2010).

[22] F. Glover, L.H. Cox, R. Patil, J.P. Kelly, Integrated exact, hybrid and metaheuristic learning methods for confidentiality protection, Annals of Operations Research, 183, 47-73 (2011).

[23] J.A. González, J. Castro, A heuristic block coordinate descent approach for controlled tabular adjustment, Computers \& Operations Research, 38, 1826-1835 (2011).

[24] M.S. Hernández, J.J. Salazar, Enhanced controlled tabular adjustment, Computers \& Operations Research, 43, 61-67 (2014).

[25] A. Hundepool, The Argus software in CENEX, Lecture Notes in Computer Science, 4302, 334-346 (2006).

[26] A. Hundepool, J. Domingo-Ferrer, L. Franconi, S. Giessing, R. Lenz, J. Naylor, E. Schulte Nordholt, G. Seri, P.-P. De Wolf, Handook on Statistical Disclosure Control v1.2, Network of Excellence in the European Statistical System in the field of Statistical Disclosure Control, 2010. Available online at http://neon.vb.cbs.nl/casc/handbook.htm

[27] A. Hundepool, J. Domingo-Ferrer, L. Franconi, S. Giessing, E. Schulte Nordholt, K. Spicer, P.-P. De Wolf, Statistical Disclosure Control. Chichester, Wiley, 2012.

[28] T. Koch, et al., MIPLIB 2010. Mixed integer programming library version 5, Mathematical Programming Computation, 3, 103-163 (2011).

[29] H. Mittelmann, Decision tree for optimization software, http://plato.asu.edu/guide.html (2014).

[30] K. Soininvaara, T. Oinonen, A. Nissinen, Balancing confidentiality and usability: protecting sensitive data in the case of inward Foreign AffiliaTes Statistics (FATS), Lecture Notes in Computer Science, 8744, 338-349 (2014).

[31] L. Zayatz, U.S. Census Bureau, communication at Joint UNECE/Eurostat Work Session on Statistical Data Confidentiality, Bilbao (Basque Country, Spain) (2009). 